ARTICLE

\title{
Mechanistic insights into the R-loop formation and cleavage in CRISPR-Cas12i1
}

Bo Zhang (1) ${ }^{1,2}$, Diyin Luo ${ }^{1,2}, Y u \mathrm{Li}^{1,2}$, Vanja Perčulija (1) ${ }^{1}$, Jing Chen ${ }^{1}$, Jinying Lin ${ }^{1}, Y_{\text {angmiao }} \mathrm{Ye}^{1} \&$ Songying Ouyang (10) ${ }^{1 凶}$

Cas12i is a newly identified member of the functionally diverse type $V$ CRISPR-Cas effectors. Although Cas12i has the potential to serve as genome-editing tool, its structural and functional characteristics need to be investigated in more detail before effective application. Here we report the crystal structures of the Cas12i1 R-loop complexes before and after target DNA cleavage to elucidate the mechanisms underlying target DNA duplex unwinding, R-loop formation and cis cleavage. The structure of the R-loop complex after target DNA cleavage also provides information regarding trans cleavage. Besides, we report a crystal structure of the Cas12i1 binary complex interacting with a pseudo target oligonucleotide, which mimics target interrogation. Upon target DNA duplex binding, the Cas12i1 PAM-interacting cleft undergoes a remarkable open-to-closed adjustment. Notably, a zipper motif in the Helical-I domain facilitates unzipping of the target DNA duplex. Formation of the 19-bp crRNA-target DNA strand heteroduplex in the R-loop complexes triggers a conformational rearrangement and unleashes the DNase activity. This study provides valuable insights for developing Cas12i1 into a reliable genome-editing tool.

\footnotetext{
${ }^{1}$ The Key Laboratory of Innate Immune Biology of Fujian Province, Provincial University Key Laboratory of Cellular Stress Response and Metabolic Regulation, Biomedical Research Center of South China, Key Laboratory of OptoElectronic Science and Technology for Medicine of Ministry of Education, College of Life Sciences, Fujian Normal University, Fuzhou, China. ${ }^{2}$ These authors contributed equally: Bo Zhang, Diyin Luo, Yu Li. ${ }^{凶}$ email: ouyangsy@fjnu.edu.cn
} 
C RISPR-Cas (clustered regularly interspaced short palindromic repeats and CRISPR-associated proteins) adaptive immune systems are used by bacteria and archaea for protection against phages and foreign mobile genetic elements $(\mathrm{MGEs})^{1-3}$. CRISPR stores information of invading nucleic acid fragments between two repeat regions of CRISPR array as spacer sequences. CRISPR-Cas systems complete their defense tasks through a three-step process of spacer acquisition, CRISPR RNA (crRNA) biogenesis, and target interference ${ }^{4,5}$. CRISPR-Cas systems exhibit great diversity and are categorized into two main classes, which are further divided into different types based on the presence of signature Cas effectors ${ }^{6-8}$. Class 1 systems (including type I, III, and IV systems) utilize multiple small Cas effectors and crRNA to form the interference module. In contrast, class 2 systems (including type II, V, and VI systems) employ a single Cas effector and crRNA for interference. Because of their simplicity and high efficiency, type II and V effectors such as Cas9, Cas12a (Cpf1), Cas12b (C2c1), and Cas12e (CasX) have been successfully developed into powerful tools for genome editing ${ }^{9-20}$.

In recent years, the search for new class 2 CRISPR-Cas systems has led to the discovery of new type $\mathrm{V}$ systems that contain Cas12c, Cas12d, Cas12g, Cas12i, Cas14a, and Cas12j effectors. These new type $\mathrm{V}$ systems are functionally distinct from the previously known class 2 effectors and can thus enrich the CRISPR-Cas toolkit for genome editing ${ }^{21-24}$. Among them, Cas12i is characterized by a relatively smaller size (1033-1093aa) compared with Cas12a. Cas12i has substantially different efficiencies in cutting two strands of target DNA, which results in a nicking activity that could be utilized to develop a double-nicking approach for genome editing with high specificity ${ }^{22,25}$. It has been reported that Cas12i has distant similarity to Cas $12 \mathrm{~b}$ and both effectors recognize a $5^{\prime}$-TTN-3' PAM (protospacer adjacent motif). Interestingly, Cas12i autonomously processes precursor crRNA (pre-crRNA) to form mature crRNA, but Cas12b itself does not own this capability ${ }^{26}$.

More recently, cryo-EM and crystal structures of Cas12i in crRNA-bound and crRNA-target-bound states have been reported $^{27,28}$. However, a number of key questions concerning the target DNA duplex unwinding, R-loop formation, target DNA duplex cleavage, key residues responsible for PAM determination in Cas12i1, and the mechanism of the DNase activation remain unanswered or require further investigation. To address these questions, we report the crystal structures of the Cas12i1 ternary complexes in the R-loop states before and after target DNA cleavage. Furthermore, we report the crystal structure of the Cas12i1 binary complex, which contains a relatively flexible PAM-interacting (PI) domain. The conformational rearrangements between the binary and ternary complexes unveil how target DNA binding triggers the DNase activity of Cas12i1. Using structural analysis and biochemical experiments, critical motifs and residues related to the aforementioned questions are identified and validated. Last, we propose a multi-step model of Cas12i1 fulfilling its function. The results of this study will provide mechanistic guidance for rational employing the CRISPRCas12i1 system for future applications in genome editing.

\section{Results}

Overall architectures of two ternary complexes of Cas12i1. To investigate how Cas12i1 recognizes, unwinds, and cleaves two strands of target DNA, we have solved two crystal structures of Cas12i1 ternary complexes. The first ternary complex, which was formed with the catalytically inactive Cas12il D647A mutant, represents the R-loop state before target DNA cleavage (hereafter termed the pre-cleavage R-loop complex) and its crystal structure was determined at $2.75 \AA$ resolution (Supplementary Table 1).
The second ternary complex uses the wild-type Cas12i1 to form the R-loop complex after target DNA cleavage (hereafter termed the post-cleavage R-loop complex) and its crystal structure was determined at $2.45 \AA$ resolution. To obtain these crystal structures, the crystal structure of the selenomethionine (SeMet)labeled wild-type Cas12il ternary complex was solved using the single-wavelength anomalous diffraction method. Next, the SeMet-labeled model was used to solve the crystal structures of two unlabeled ternary complexes using the molecular replacement method. The asymmetric unit of both crystal structures contains a single ternary complex. The structures of Cas12i1 in two ternary complexes resemble each other, and the root-meansquare deviation (RMSD) value for their backbone Ca atoms is $0.52 \AA$. Major differences between the two complexes exist in the states of target DNA, which will be described in depth later.

Compared with the other type $\mathrm{V}$ effectors with known structures, the structure of Cas12i1 in the ternary complex displays significant structural divergence. The typical bilobed architecture consisting of the recognition (REC) lobe and the nuclease (NUC) lobe can be easily distinguished, though organization and distribution of individual domains in Cas12i1 are distinct from those of Cas12a, Cas12b, and Cas12e $20,29-33$ (Fig. 1a-e and Supplementary Fig. 1a-d). In Cas12i1, the REC lobe consists of the Helical-I domain and the PAM-interacting (PI) domain, whereas the NUC lobe comprises the Wedge (WED), Helical-II, RuvC, and Nuc domains (Fig. 1a, d and Supplementary Fig. 2a-g). The mature crRNA and target DNA are sandwiched inside the positively charged pocket formed by the two lobes (Fig. 1b-e). To be noted, two separate $a$-helix bundles in the Helical-I domain are located at the PAM-proximal and PAM-distal regions, respectively, which allows the Helical-I domain to extend over the long axis of the whole ternary complex (Supplementary Fig. 2a, b).

A mature 43-nt crRNA consisting of 23-nt repeat region and 20-nt guide region was determined in both ternary complexes. The crRNA originated from a co-expressed CRISPR array template and was processed by the expressed Cas12i1 in E. Coli cells. Overall, the crRNA repeat region approximately adopts a stem-loop structure, and the R-loop formed by crRNA and target DNA in the pre-cleavage R-loop complex displays a T-shaped architecture (Fig. 1c and Supplementary Fig. 3a). The nucleotides $\mathrm{U}(1)-\mathrm{A}(19)$ of the crRNA guide region and $\mathrm{dA}(1)-\mathrm{dT}(19)$ of the target DNA (TD) strand form a 19-bp heteroduplex. The nucleotides $\mathrm{dG}(-1)-\mathrm{dC}(-9)$ of the $\mathrm{TD}$ strand and $\mathrm{dC}$ $\left(-1^{*}\right)-\mathrm{dG}\left(-9^{*}\right)$ of the non-target DNA (NTD) strand form the PAM duplex (Fig. 1b, c).

Recognition of the crRNA repeat region. The extensive interactions between the stem-loop structure of the crRNA repeat region and Cas12il are mainly involved in stabilizing the sugarphosphate backbone of the stem-loop structure (Supplementary Figs. $4 \mathrm{a}, \mathrm{b}$ and 5 ). The short helix $\mathrm{a} 2$ of the WED domain is located above the $\mathrm{G}(-17): \mathrm{C}(-1)$ base pair of the stem duplex, which markedly distorts orientations of the nucleotides $\mathrm{U}(-19)$ and $\mathrm{U}(-18)$ (Supplementary Fig. 4c). Consequently, the $5^{\prime}$ unpaired repeat region nucleotides $\mathrm{A}(-23)-\mathrm{U}(-18)$ swing away from the stem duplex and extend into the pre-crRNA processing pocket within the WED domain. Particularly, the nucleotide A (-23) stacks with residues H497 and H528 (Supplementary Fig. 4c). The nucleotide U(-22) stacks with the side chain of R503 and forms hydrogen bonds with K494 and D507. The nucleotide $\mathrm{U}(-21)$ stacks with Y509 and hydrogen bonds with W511. The nucleotide $\mathrm{U}(-18)$ hydrogen bonds with Y509 and the backbone oxygen atom of G584. Details of the interactions between Cas12i1 and the crRNA are presented in Supplementary Fig. 5. 
a

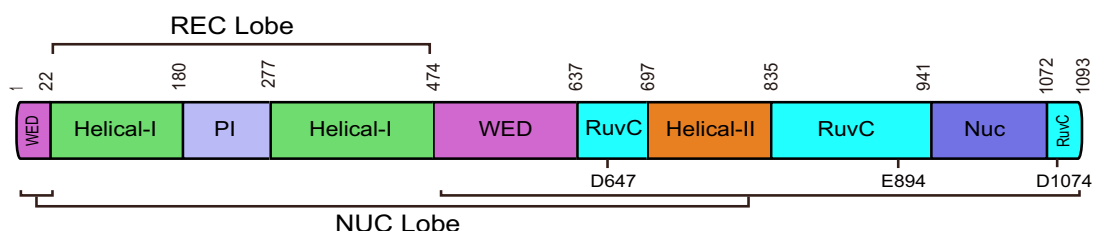

b

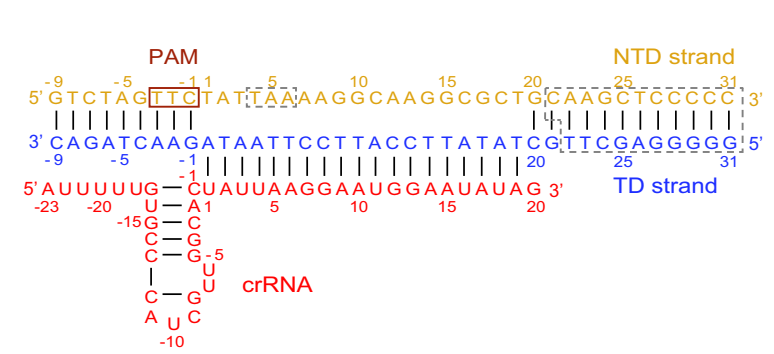

C

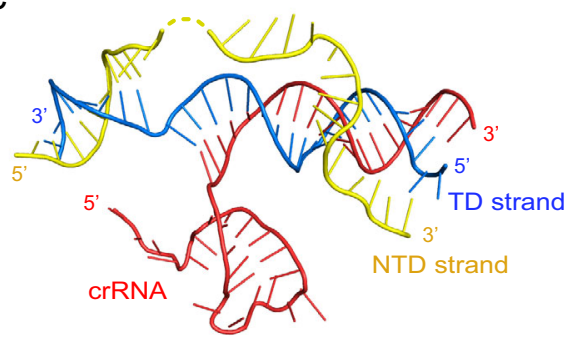

d
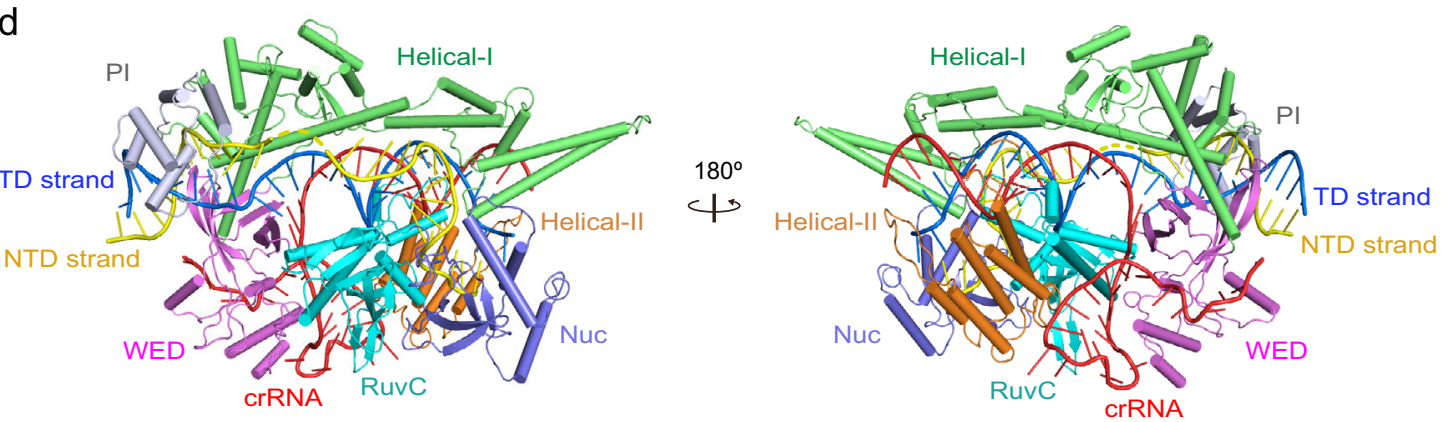

e
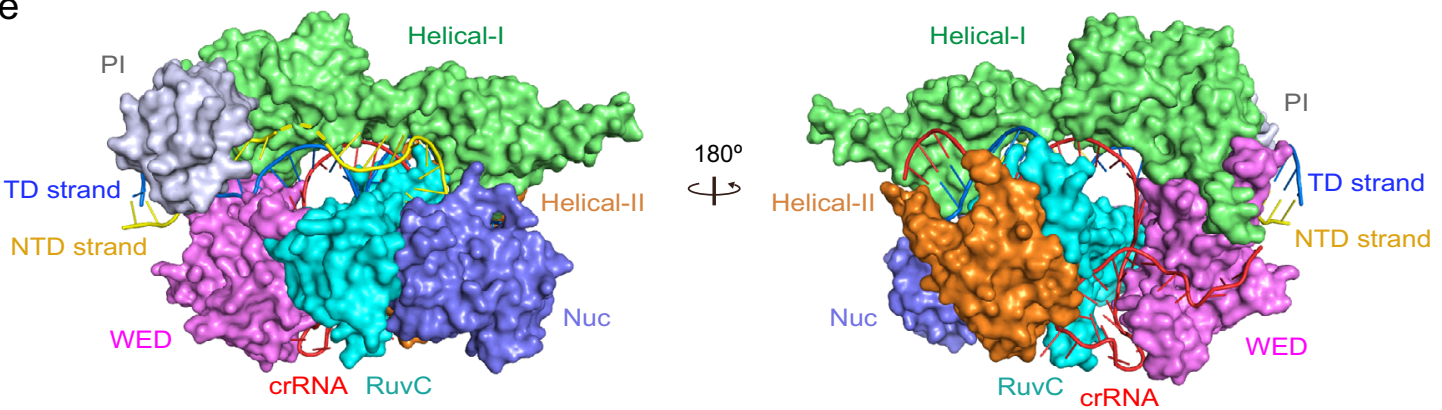

Fig. 1 Crystal structure of the Cas12i1 pre-cleavage R-loop complex. a Domain organization of Cas12i1. Catalytic residues within the RuvC domain are annotated below the diagram. $\mathbf{b}$ Schematic representation of the R-loop structure formed by crRNA and the target duplex. Disordered regions are surrounded by gray dashed lines. c View of the R-loop structure in the complex. d Overall structure of the Cas12i1 pre-cleavage R-loop complex shown in two different orientations and color-coded as defined in (a) and (b). e Surface representations of the Cas12i1 pre-cleavage R-loop complex shown in the same views as in (d).

Mechanism of the $5^{\prime}$-TTN-3' PAM determination. The PAM duplex is gripped inside a positively charged cleft formed by the Helical-I, PI, and WED domains (Fig. 2a, b). The PI domain is connected to the Helical-I domain via two loops, which would provide the PI domain with remarkable flexibility prior to binding the PAM duplex. In pre-cleavage R-loop complex, a loop between the helices $a 3$ and $a 4$ of the PI domain inserts into the minor groove of the PAM duplex to contact the PAM nucleotides dT $\left(-3^{*}\right)$ and $\mathrm{dT}\left(-2^{*}\right)$ in the NTD strand (Fig. 2a). Concurrently, another loop between the strands $\beta 2$ and $\beta 3$ of the WED domain inserts into the major groove of the PAM duplex and interacts with the PAM-complementary nucleotides $\mathrm{dA}(-3)-\mathrm{dG}(-1)$ in the TD strand. The $5^{\prime}-\mathrm{TTN}-3^{\prime}$ PAM is recognized mainly by hydrogen bonds. Firstly, the $\mathrm{O} 4$ atom of $\mathrm{dT}\left(-3^{*}\right)$ hydrogen bonds with the side chain of $\mathrm{H} 170$ and the 5-methyl group of $\mathrm{dT}\left(-3^{*}\right)$ engages in the van der Waals interaction with the side chain of
L298 (Fig. 2c). The N6 and N7 atoms of $\mathrm{dA}(-3)$ form hydrogen bonds with the side chain of N481 (Fig. 2d). Notably, the Ca atom of residue G235 is adjacent to the nucleobases of the $\mathrm{dA}(-3): \mathrm{dT}$ $\left(-3^{*}\right)$ base pair, suggesting that any substitution of G235 would directly disrupt the correct recognition of this base pair. Modeling of the $\mathrm{dG}(-3): \mathrm{dC}\left(-3^{*}\right)$ base pair to replace the $\mathrm{dA}(-3): \mathrm{dT}\left(-3^{*}\right)$ base pair would generate a steric clash between the N2 atom of dG $(-3)$ and the backbone nitrogen atom of residue A236 (Supplementary Fig. 6a). Secondly, the $\mathrm{O} 2$ atom of $\mathrm{dT}\left(-2^{*}\right)$ hydrogen bonds with the backbone nitrogen atom of A236 (Fig. 2c). The N6 and N7 atoms of $\mathrm{dA}(-2)$ form hydrogen bonds with the side chain of S482 (Fig. 2d). Modeling of the $\mathrm{dG}(-2): \mathrm{dC}\left(-2^{*}\right)$ base pair to replace the $\mathrm{dA}(-2): \mathrm{dT}\left(-2^{*}\right)$ base pair would also generate steric clashes between the $\mathrm{N} 2$ atom of $\mathrm{dG}(-2)$ and the side chain of A236 (Supplementary Fig. 6b), thus explaining the requirement of double $\mathrm{T}$ in the PAM. Thirdly, the $\mathrm{O} 6$ atom of $\mathrm{dG}(-1)$ forms a 

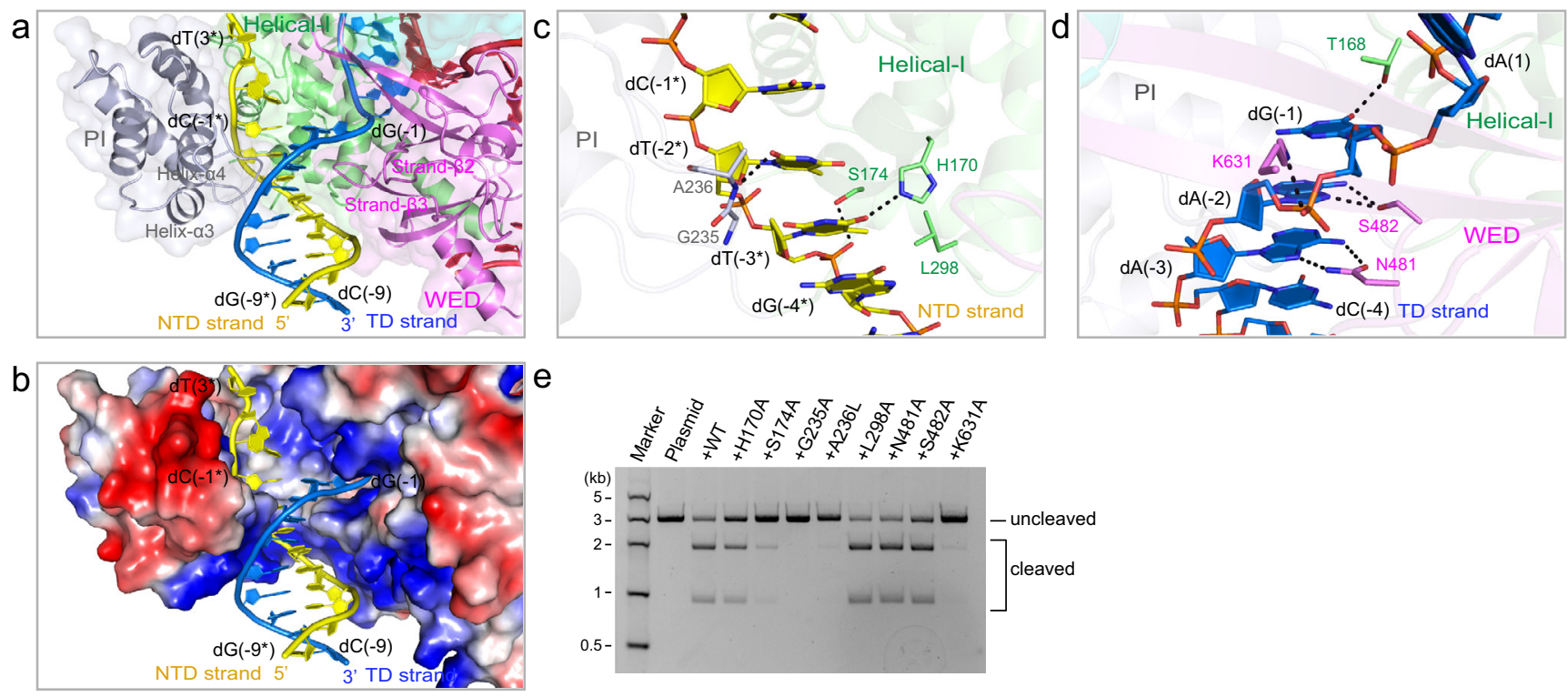

Fig. 2 Recognition of the PAM duplex. a Recognition of the PAM duplex in the Cas12i1 pre-cleavage R-loop complex and the PAM-interacting cleft formed by the Helical-I, PI, and WED domains. The loop between strands $\beta 2$ and $\beta 3$ of the WED domain inserts into the major groove of the PAM duplex, and another loop between helices $\alpha 3$ and $\alpha 4$ in the PI domain inserts into the minor groove of the PAM duplex. $\mathbf{b}$ Electrostatic potential surface of the PAMinteracting cleft. Red, white, and blue indicate negative, neutral, and positive electrostatic potential surfaces, respectively. c Recognition of the NTD strand in the PAM duplex. $\mathbf{d}$ Recognition of the TD strand in the PAM duplex. e Agarose gel demonstrating the cleavage of the linearized plasmid by wild-type Cas12i1 and the mutants in complex with crRNA. The cleavage assays were repeated three times independently to confirm the repeatability and source data are provided as a Source Data file.

hydrogen bond with the side chain of T168 (Fig. 2d). It lacks specific recognition at this position in the PAM. Aside from the aforementioned interactions, a number of other Cas12il residues play a role in stabilizing the sugar-phosphate backbone of the PAM duplex (Supplementary Fig. 5). The results of the cleavage assays show that the previously unreported G235A and A236L mutations almost abolished the cleavage activity. The S174A and K631A mutations decrease this activity (Fig. 2e). Therefore, residues G235 and A236 play a critical role in PAM determination.

Mechanism of the R-loop formation in Cas12il. In the precleavage R-loop complex, the two strands of target DNA are unwound beyond the PAM duplex and the TD strand forms a 19bp heteroduplex with the crRNA guide region. The PAMproximal region of the heteroduplex contacts the Helical-I, WED, and RuvC domains, and the PAM-distal region of the heteroduplex interacts with the Helical-I, Helical-II, and RuvC domains (Fig. 1d). The NTD strand extends from the PAM duplex and is partially exposed to the surrounding solvent. The nucleotides $\mathrm{dT}$ $\left(4^{*}\right)-\mathrm{dA}\left(6^{*}\right)$ of the NTD strand are disordered and invisible due to the flexibility, and the nucleotides $\mathrm{dA}\left(7^{*}\right)-\mathrm{dG}\left(20^{*}\right)$ pass through the channel formed by the Helical-I, Helical-II, RuvC, and Nuc domains. Finally, the nucleotide dG(20*) of the NTD strand interacts with $\mathrm{dC}(20)$ of the TD strand (Fig. 1c, d).

Notably, helix $\alpha 7$ and a loop region between helices $\alpha 6$ and $\alpha 7$ of the Helical-I domain are positioned to obstruct the possible dA (1)-dA(3): $\mathrm{dT}\left(1^{*}\right)-\mathrm{dT}\left(3^{*}\right)$ base pairings and prompt the flipping of $\mathrm{dA}(1)-\mathrm{dA}(3)$, thus behaving as a "zipper" for unwinding of the target DNA duplex, and are henceforth referred to as the zipper motif (residues K160-F177) (Fig. 3a). To investigate the function of the zipper motif, we generated a protein construct in which the loop region (residues K160-A169) of the zipper motif was replaced by a simple linker (residues GGSGGS). When cleaving DNA substrates containing 1-4-bp mismatched bubbles adjacent to the PAM, the $\triangle 160-169$ aa construct show the decreased cleaving capacity (Fig. 3b). The cleavage activity was restored by increasing the length of mismatched bubbles, which denotes that the zipper motif plays an important role for facilitating the target duplex unwinding. Therefore, binding of the target duplex and subsequent recognition of the correct PAM sequence by Cas12i1 could make the PAM-proximal region of the target duplex approach the zipper motif, and the zipper motif could generate a spatial obstruction toward base pairing within the PAM-proximal region of the target duplex and contribute to the nucleation between the TD strand and the seed region of the crRNA guide.

Besides the zipper motif, other regions or residues of Cas12i1 also play a role in the target duplex unwinding. Helix $\alpha 2$ and strand $\beta 1$ of the WED domain are arranged above the stem duplex of the crRNA repeat region (Fig. 3c). They mediate a flipping of the nucleotide $\mathrm{U}(1)$ relative to $\mathrm{C}(-1)$ in the crRNA and facilitate base pairing between $\mathrm{U}(1)$ of the crRNA and $\mathrm{dA}(1)$ of the TD strand. More specifically, the side chain of R535 stacks with the pyrimidine ring of $\mathrm{C}(-1)$, while residues $\mathrm{R} 535$ and $\mathrm{K} 923$ form salt bridges with the phosphate group between $\mathrm{U}(1)$ and $\mathrm{C}(-1)$, which profoundly shapes the orientation of $\mathrm{U}(1)$ in the crRNA. Residues R535 and K13 interact with E539 to further stabilize the orientation of U(1). Concurrently, the side chain of R12 stacks with the purine ring of $\mathrm{dA}(1)$ in the TD strand, and residues R12 and $\mathrm{K} 483$ form salt bridges with the phosphate group between $\mathrm{dA}$ (1) and $d G(-1)$. These interactions facilitate the base pairing between $\mathrm{dA}(1)$ of the TD strand and U(1) of the crRNA. Such kind of "phosphate lock" interactions has also been found in Cas9, Cas12a, and Cas12b $\mathrm{b}^{29,33-37}$. The mutational studies show that the R535A mutation greatly decreases the cleavage activity, and the R12A, K483A, and K13A mutations slightly decrease this activity (Fig. 3d). Altogether, two groups of "phosphate lock" interactions, one group (residues R535 and K923) interacting with the crRNA and the other group (residues R12 and K483) interacting with the TD strand, have been identified in Cas12il to facilitate the nucleation between the TD strand and the crRNA guide.

The crRNA guide-TD strand heteroduplex is flanked by Cas12i1 on both sides throughout its length. The long helix a9 

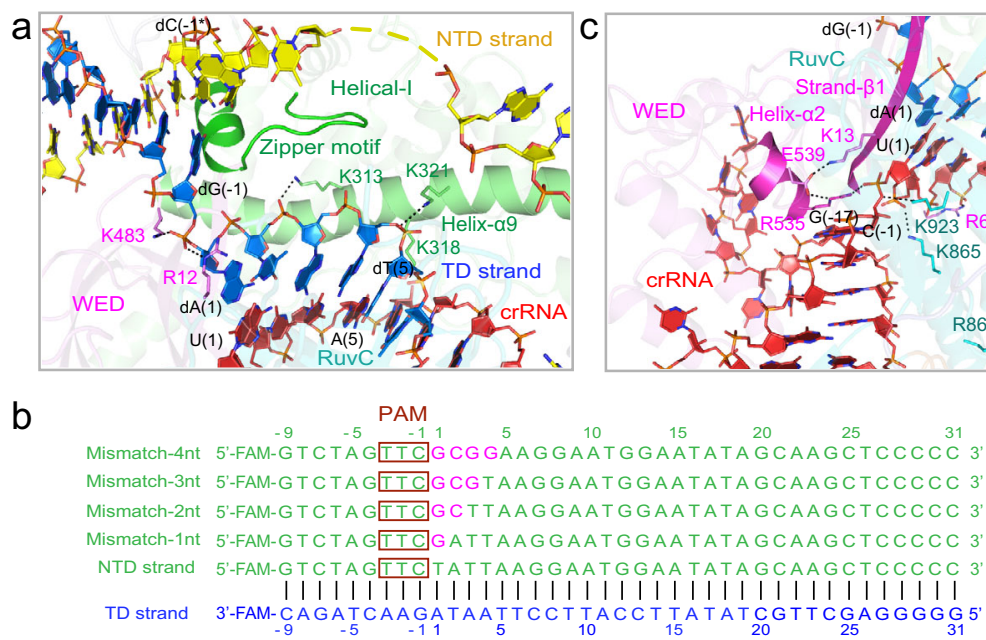

b

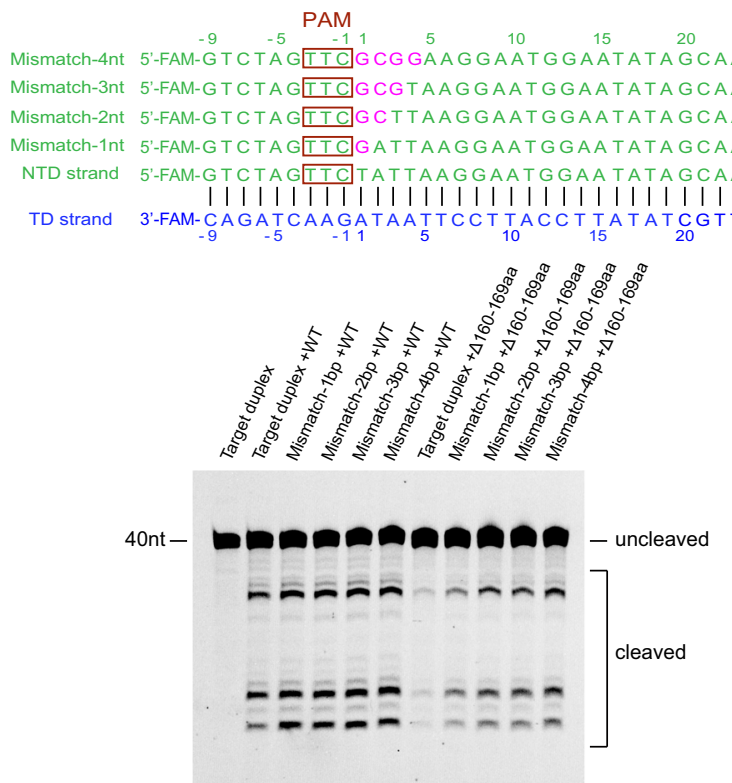

d

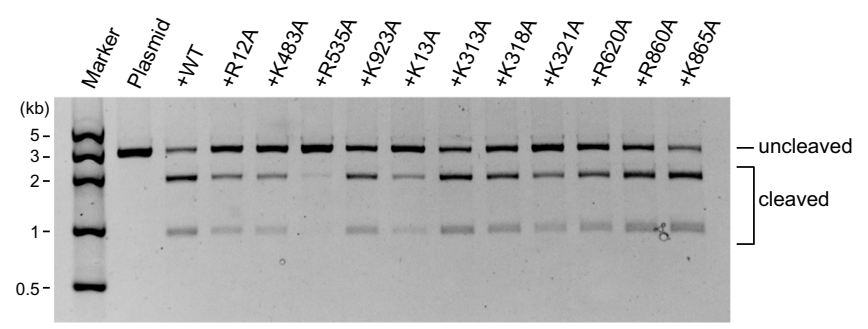

e

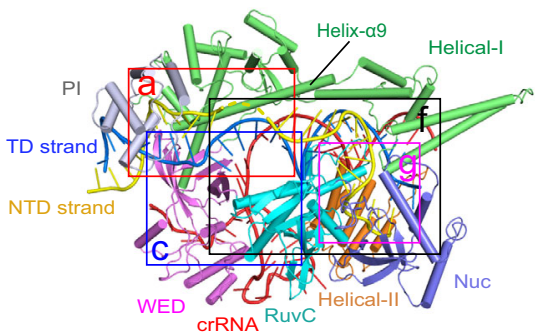

f

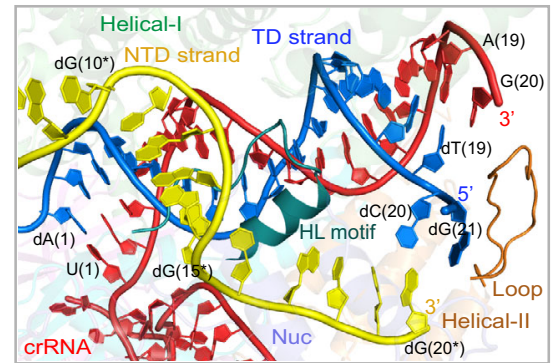

g

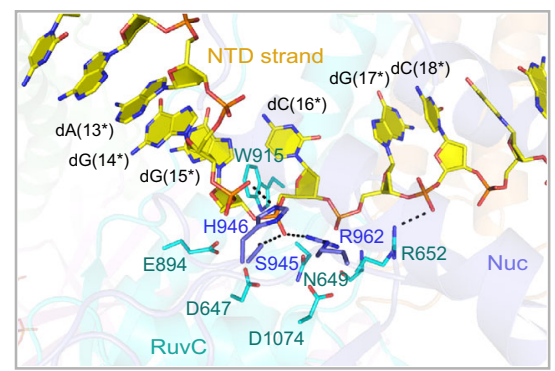

$\mathrm{h}$

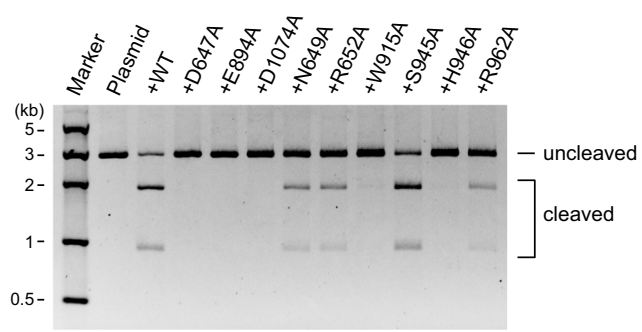

Fig. 3 The target duplex unwinding and R-loop formation. a Detailed interactions between the unzipped target duplex and Cas12i1 at the PAM-proximal region of the Cas12i1 pre-cleavage R-loop complex. The zipper motif (residues 160-177aa) facilitates unwinding of the target duplex. $\mathbf{b}$ Schematic representations of the fluorescently labeled target duplex and 1-4-nt mismatched NTD strands designed to form 1-4-bp mismatched duplexes. Denaturing gel demonstrating the cleavage of the fluorescently labeled target duplex or mismatched duplexes by wild-type Cas12i1 and the $\triangle 160-169$ aa mutant in complex with crRNA. c Detailed interactions between the PAM-proximal region of the heteroduplex and Cas12i1. $\mathbf{d}$ Agarose gel demonstrating the cleavage of the linearized plasmid by wild-type Cas12i1 and the mutants in complex with crRNA. e The relative positions of (a), (c), (f), and (g) in the Cas12i1 precleavage R-loop complex. The long helix $\alpha 9$ of the Helical-I domain is labeled. $\mathbf{f}$ The PAM-distal region of the R-loop structure. The HL motif (residues 895-915aa) of the RuvC domain is located between the crRNA guide region-TD strand heteroduplex and the unpaired NTD strand. A loop region of the Helical-II domain helps the TD strand interacting with the NTD strand. $\mathbf{g}$ Detailed interactions between the unpaired NTD strand and the RuvC catalytic pocket of Cas12i1. Residue A647 of the catalytically inactive Cas12i1 pre-cleavage R-loop complex was virtually mutated back to D647. $\mathbf{h}$ Agarose gel demonstrating the cleavage of the linearized plasmid by wild-type Cas12i1 and the mutants related to the RuvC catalytic pocket in complex with crRNA. The cleavage assays of (b), (d), and (h) were repeated three times independently to confirm the repeatability, respectively, and source data are provided as a Source data file.

of the Helical-I domain stretches along one flank of the heteroduplex (Fig. 3a, e). Residues K313, K318, and K321 of helix a9 stabilize the sugar-phosphate backbone of the PAMproximal region in the TD strand (Fig. 3a). On the other flank of the heteroduplex, a helix-loop (HL) motif (residues N895-W915) in the RuvC domain acts as a guardrail that restricts the unpaired region of the NTD strand and the heteroduplex to two separate channels (Fig. 3f). The heteroduplex-binding channel is formed by the Helical-I, Helical-II, and RuvC domains, whereas the NTD strand-binding channel is formed by the Helical-I, Helical-II, RuvC, and Nuc domains. Single mutations to alanine of residues $\mathrm{K} 313, \mathrm{~K} 318, \mathrm{~K} 321, \mathrm{R} 620$, R860, and K865 interacting with the heteroduplex have minor effects on the cleavage activity (Fig. 3a, c, d). At the PAM-distal end of the pre-cleavage R-loop complex, a loop region (residues N724-I737) between helices $\alpha 1$ and $\alpha 2$ of the Helical-II domain impedes base pairing between $\mathrm{dC}(20)$ and $\mathrm{G}(20)$ (Fig. 3f). Consequently, the nucleotides $\mathrm{dC}(20)$ and $\mathrm{dG}(21)$ of the TD strand make a sharp turn relative to $\mathrm{dT}(19)$, which 
facilitates $\mathrm{dC}(20)$ interacting with $\mathrm{dG}\left(20^{*}\right)$. Due to the flexibility or instability, the target duplex beyond the $\mathrm{dC}(20): \mathrm{dG}\left(20^{*}\right)$ base pair is hardly distinguishable in the present model.

The structure of the pre-cleavage R-loop complex displays how the NTD strand is accommodated within the RuvC active site (Fig. 3g). Residue W915 intervenes between $\mathrm{dG}\left(15^{*}\right)$ and $\mathrm{dC}\left(16^{*}\right)$, and affects the orientation of $\mathrm{dC}\left(16^{*}\right)$. Residue H946 interacts with the phosphate group of $\mathrm{dG}\left(15^{*}\right)$. W915 and H946 work together to restrict the sugar-phosphate backbone of the NTD strand passing through the RuvC active site. Side chains of three catalytic residues D647 (virtual modeling of A647D), E894, and D1074 in the RuvC domain point toward the phosphate group between the nucleotides $\mathrm{dG}\left(15^{*}\right)$ and $\mathrm{dC}\left(16^{*}\right)$, implying this position as the possible cutting site in the NTD strand. Moreover, residues S945 and R962 interact with the phosphate group of dC $\left(16^{*}\right)$, and R652 forms a salt bridge with the phosphate group of $\mathrm{dC}\left(18^{*}\right)$. The results of the cleavage assays show that the D647A, E894A, and D1074A mutants are catalytically inactive, the W915A and $\mathrm{H} 946 \mathrm{~A}$ mutants display near-complete loss of the cleavage activity, and the N649A, R652A, and R962A mutants have a decreased cleavage activity (Fig. $3 \mathrm{~h}$ ).

The sequential cis-cleavage of the NTD and TD strands. To investigate the sites at which Cas12il cuts two strands of the target duplex, the 40-nt NTD and TD strands were labeled with two different dyes and annealed (Fig. 4a). The cleavage assay shows that the NTD strand was successively cleaved at 13-15 nucleotides after the PAM duplex, whereas the TD strand was cleaved at 24 nucleotides after the PAM duplex (Fig. 4b). Given that the unpaired region of the NTD strand preoccupies the RuvC active site of the pre-cleavage R-loop complex, it is not difficult to understand that Cas12il cleaves the NTD strand prior to the TD strand. The sequential cis-cleavage of the NTD and TD strands has been observed or discussed in Cas12a, 12b, and 12e effectors $20,29,38,39$, and is further validated for Cas12i1 by our time-course cleavage assay (Fig. $4 \mathrm{~b}$ ).

In the post-cleavage R-loop complex, both NTD and TD strands are already cleaved by Cas12il. The PAM-distal region of the target duplex is released, and the PAM-proximal region of the target duplex is retained in the complex (Fig. 4c, d and Supplementary Fig. 3b). Interestingly, the cleaved NTD strand stretches out of Cas12il and is captured by the neighboring complex from another asymmetric unit (Fig. 4e). Correspondingly, the cleaved NTD strand of the neighboring complex is determined passing through the RuvC catalytic pocket of the post-cleavage R-loop complex. The overall conformation of the unpaired region $\mathrm{dA}\left(6^{*}\right)-\mathrm{dA}\left(13^{*}\right)$ of the NTD strand from the neighboring complex extended into the post-cleavage R-loop complex resembles that of the unpaired region $\mathrm{dC}\left(11^{*}\right)-\mathrm{dC}\left(18^{*}\right)$ of the NTD strand in the pre-cleavage R-loop complex (Fig. 4f). Hence, the post-cleavage R-loop complex not only represents a state of the R-loop complex after the cis-cleavage of the target duplex, but also provides additional information about the precleavage state for the trans-cleavage.

For some unknown reason, the cleaved NTD strand accommodated within the RuvC active site was not further transprocessed in the post-cleavage R-loop complex. Notably, three water molecules were identified to form multiple hydrogen bonds within the RuvC catalytic site (Fig. 4g). Among them, one water molecule interacts with residues D647, N649, D1074 and the phosphate group of $\mathrm{dC}\left(11^{*}\right)$. Another water molecule forms hydrogen bonds with residues D647, E894, T944 and S945. Typically, the conserved RNase $\mathrm{H}$ fold utilizes a two-metal-ion catalytic mechanism for phosphoryl hydrolysis ${ }^{40,41}$. Considering that the RuvC domain in Cas12il also possesses such RNase $\mathrm{H}$ fold, positions of these water molecules in the present structure are possibly occupied by two-metal ions, which would be in accordance with the recently reported presence of two metal ions in the RuvC active site of the Cas12i2 ternary complex ${ }^{28}$.

A loop region in the Helical-II domain facilitates the TD strand loading into the RuvC active site. Following the cleavage of the NTD strand, the mechanism of the TD strand loading into the RuvC active site for cleavage remains to be clarified. The TD strand should enter the RuvC active site via a pathway surrounded by the Helical-II, RuvC, and Nuc domains. In the precleavage R-loop complex, a loop region in the Helical-II domain facilitates bending of the $5^{\prime}$-terminal of the TD strand toward the Nuc domain, in which the TD strand interacts again with the NTD strand (Fig. 5a). We substituted this loop region (residues N724-I737) or the region containing this loop and two flanking helices (residues L706-E750) with a linker (residues GGSG). The cleavage assays show that both constructs affect the trans-cleavage pattern and slightly increase the trans-cleavage activity for the NTD strand, whereas they decrease the cis-cleavage activity for the TD strand (Fig. 5b). Therefore, this loop region in the Helical-II domain facilitates the loading of the TD strand beyond the heteroduplex into the RuvC active site for cleavage.

Base pairings within the seed region mimicking the target interrogation. To understand how Cas12il recognizes mature crRNA and the conformational features prior to the target duplex binding, we solved the crystal structure of the Cas12i1-crRNA binary complex at $3.6 \AA$ resolution. The global structure of Cas12i1 in the binary complex also exhibits the bilobed architecture, and the crRNA repeat region is recognized by Cas12i1 in the manner observed in the R-loop complexes (Fig. 6a). Nucleotides U(1)-A (15) of the crRNA guide region were identified in the binary complex. The first six nucleotides $\mathrm{U}(1)-\mathrm{A}(6)$ adopt a preorganized and nearly A-form helical conformation (Fig. 6b). In Cas9, Cas12a, and Cas $12 \mathrm{~b}$, the preorganized PAM-adjacent seed region within the crRNA guide region is essential for target recognition $29,42,43$. The seed region of the Cas12il crRNA is approximately within the first seven nucleotides on the $5^{\prime}$ end of the crRNA guide region ${ }^{27}$. Interestingly, the seed nucleotides $\mathrm{A}(2)-\mathrm{U}(4)$ in the present study pair with a complementary 3 -nt oligonucleotide that originated from E. coli cells and was captured by the binary complex (Fig. 6a, b and Supplementary Fig. 3c). Besides, the other seed nucleotides $\mathrm{U}(1)$ and $\mathrm{A}(5)-\mathrm{A}(6)$ maintain a preordered conformation. These base pairings within the seed region mimic the scene of target interrogation by the Cas12i1-crRNA interference module.

Target binding activates the Cas12il DNase activity. In the binary complex, the PI domain protrudes from the main body of Cas12il and extends into the surrounding solvent (Fig. 6a). Due to the flexibility, a part of the PI domain including helix $\alpha 3$ is invisible. An overlay of the binary and ternary complexes reveals that the PAM-interacting cleft undergoes an "open to closed" conformational transition to clench the PAM duplex (Fig. 6c-e). Inside the PI domain, binding to the PAM duplex induces conformational adjustment of helices $\alpha 2$ and $\alpha 4$ relative to helices $\alpha 1$ and $\alpha 5$. Thus, two key features of the resting state of the Cas12i1crRNA binary complex that poise it for the target duplex binding are the open PAM-interacting cleft and preorganized seed region.

The overlay also indicates that the Helical-I domain exhibits an apparent rearrangement, whereas the NUC lobe as a whole makes a slight rearrangement (Fig. 6c, d). In the binary complex, the RuvC active site is shielded from substrate binding by the PAMdistal $\alpha$-helix bundle of the Helical-I domain and the HL motif of the RuvC domain (Fig. 6f, g). Besides, Cas12il arranges its 
a

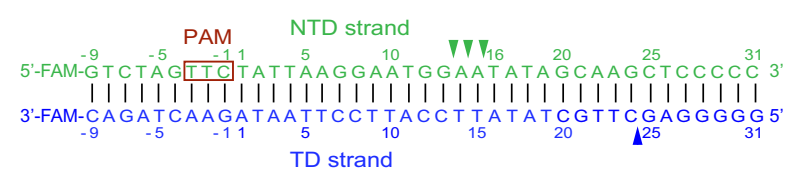

C

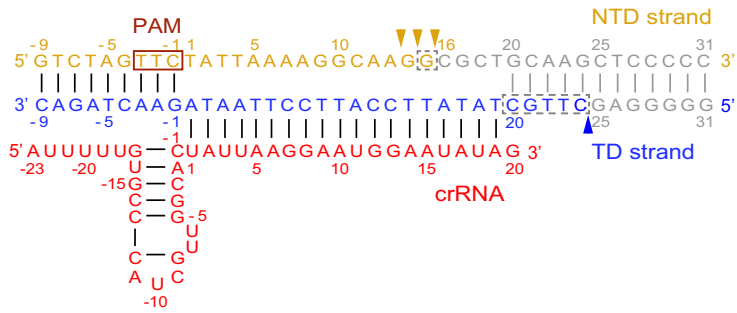

b
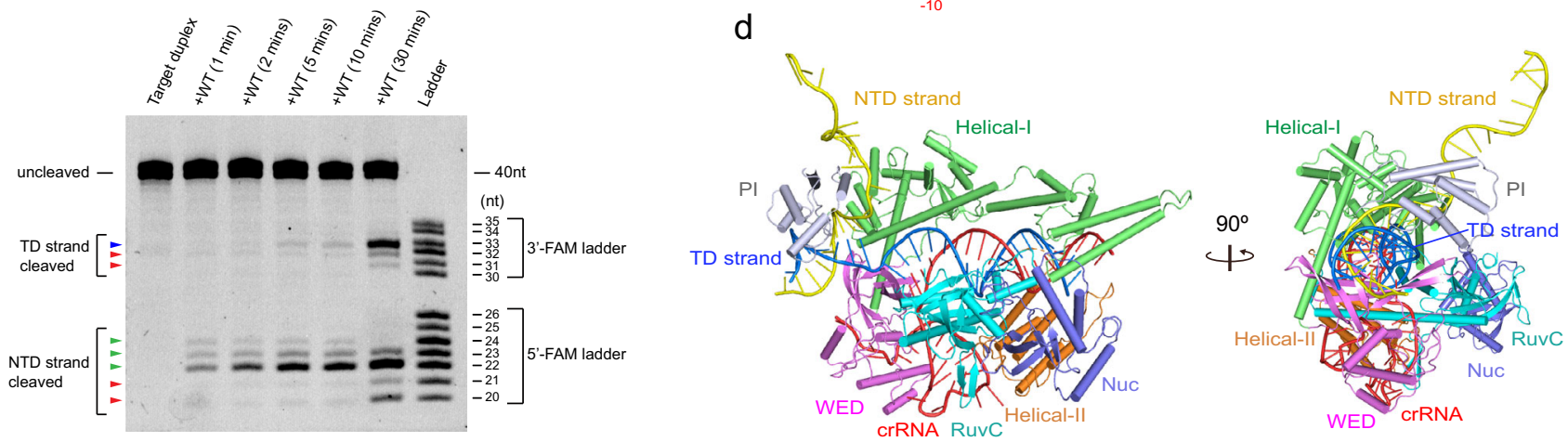

e

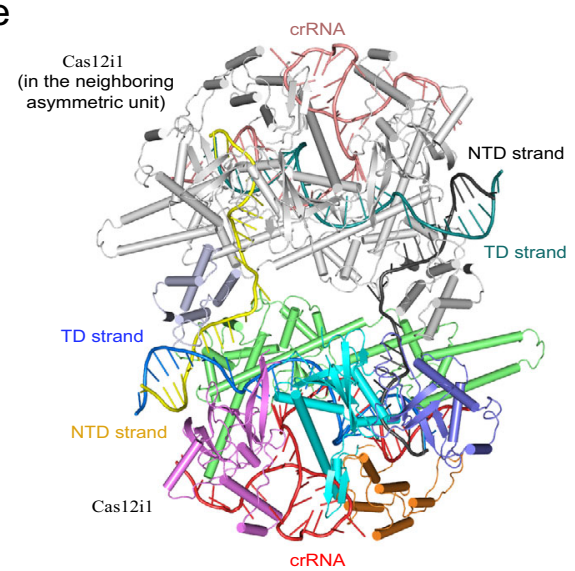

f

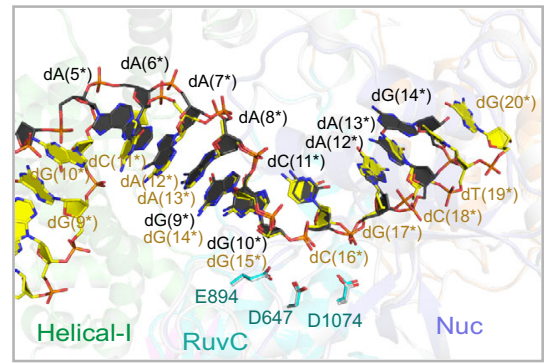

g

Fig. 4 Crystal structure of the Cas12i1 post-cleavage R-loop complex. a Schematic representations of the fluorescently labeled target duplex used in the cleavage assays. Green and blue triangles indicate the cleavage sites within the NTD and TD strands, respectively. b Denaturing gel demonstrating the time-course cleavage of the fluorescently labeled target duplex by wild-type Cas12i1 in complex with crRNA. The cis-cleavages of the NTD and TD strands are labeled with the green and blue triangles, respectively, and the trans-cleavages are labeled with the red triangles. The cleavage assays were repeated three times independently to confirm the repeatability and source data are provided as a Source data file. c Schematic representation of the R-loop structure after target DNA cleavage. Yellow and blue triangles indicate the cleavage sites within the NTD and TD strands, respectively. Nucleotides colored in gray represent the PAM-distal cleavage product that was released from the complex. Disordered regions are encircled by gray dashed lines. $\mathbf{d}$ Overall structure of the Cas12i1 post-cleavage R-loop complex shown in two different orientations, color-coded as defined in Fig. 1. e The cleaved NTD strand of the Cas12i1 post-cleavage R-loop complex is extended into the RuvC active site of the neighboring complex from another asymmetric unit and vice versa. Each asymmetric unit contains one post-cleavage R-loop complex. f, The overlay of the unpaired NTD strand from the pre-cleavage R-loop complex and the unpaired NTD strand from the neighboring complex extended into the post-cleavage R-loop complex. The pre-cleavage R-loop complex is colored as in Fig. 1. The neighboring unpaired NTD strand and Cas12i1 in the post-cleavage R-loop complex are colored black and silver, respectively. $\mathbf{g}$ Detailed interactions between the neighboring unpaired NTD strand and the RuvC active site of the post-cleavage R-loop complex. The figure is colored as in (e).

multiple domains to form one central channel for binding the crRNA guide region. During transition to the ternary complex, Cas12i1 rearranges the Helical-I domain and the HL motif to form two separate channels, one for the heteroduplex and the other for the NTD strand or the trans-cleavage substrate (Fig. 6f, g). Notably, the overlay shows that the target duplex binding drives upward movement of the PAM-distal $\alpha$-helix bundle of the Helical-I domain (Fig. 6c, d). Moreover, the HL motif of the RuvC domain swings toward the center of Cas12i1 (Fig. 6f, g). As a result of these pivotal changes, the PAM-distal $\alpha$-helix bundle and the HL motif no longer obstruct the RuvC active site and the DNase activity of Cas12il is unleashed.
To understand how many base pairings between the crRNA guide region and the TD strand are required for the conformational activation of Cas12i1, we use double-stranded (ds) or single-stranded (ss) target DNA with different lengths to perform the cleavage assays. The results indicate that a 12-bp target dsDNA and a 12-nt target ssDNA can achieve detectable cleavage activity, whereas 14-16-bp target dsDNA and 13-16-nt target ssDNA can fully activate the enzyme (Fig. 6h).

The temperature and metal-ion dependent substrate cleavage. To investigate whether the substrate cleavage by Cas12i1 is a 
a

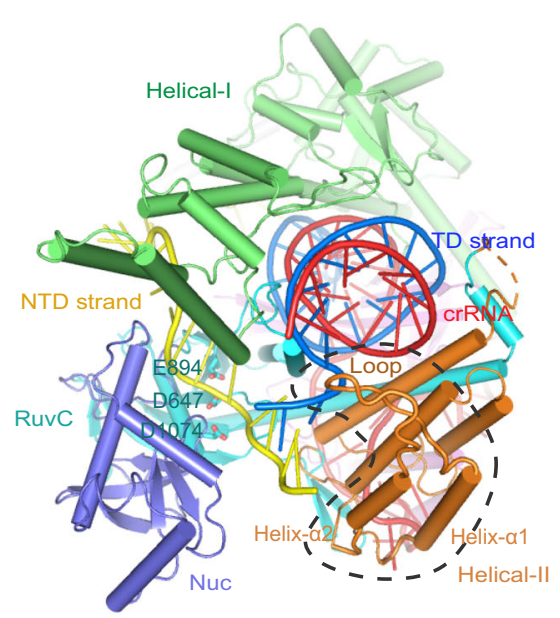

b

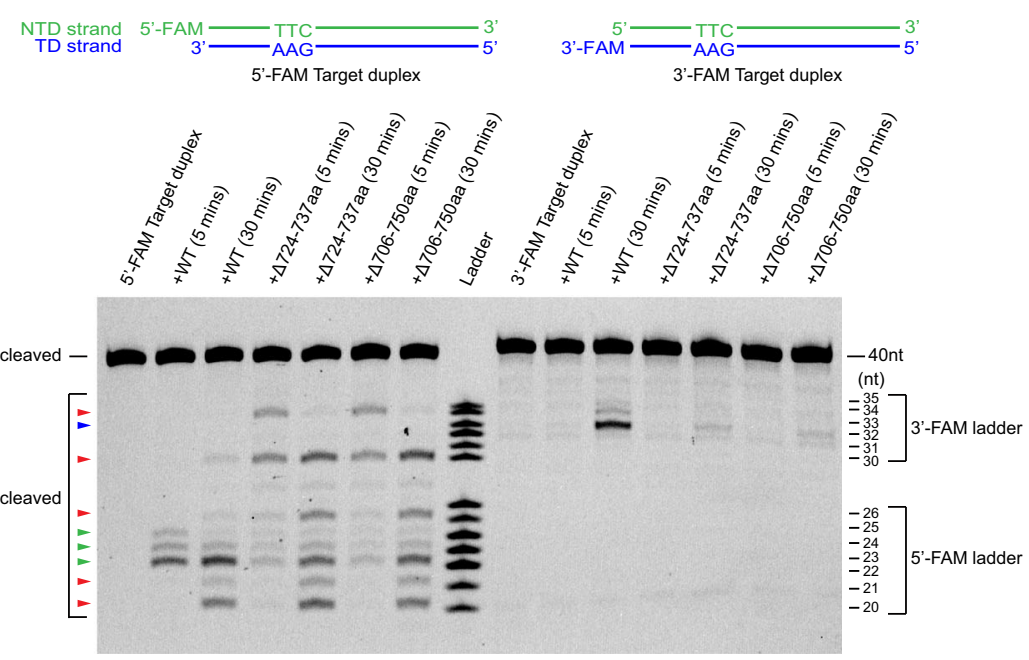

Fig. 5 A loop region in the Helical-II domain facilitating the TD strand loading. a A loop region (residues 724-737aa) and the region comprising this loop and two connected helices (residues 706-750aa) in the Cas12i1 R-loop ternary complex are encircled with a black dashed line. b Denaturing gel demonstrating the cleavage of the fluorescently 5'-FAM-NTD strand-labeled or 3'-FAM-TD strand-labeled DNA substrate by wild-type Cas12i1, $\triangle 724-737 a a$, and $\triangle 706-750$ aa mutants in complex with crRNA. The cis-cleavages of the NTD and TD strands are labeled with the green and blue triangles, respectively, and the trans-cleavages are labeled with the red triangles. The cleavage assays were repeated three times independently to confirm the repeatability and source data are provided as a Source data file.

temperature or metal-ion-dependent process, we carried out the cleavage assays at different temperatures or by adding different metal ions to the reaction mixture. The results show that Cas12i1 is capable of cleaving substrates between 37 and $57^{\circ} \mathrm{C}$, and has the favorable cleaving temperature of $45^{\circ} \mathrm{C}$ (Supplementary Fig. 7a). Moreover, among the tested divalent metal ions, only $\mathrm{Mg}^{2+}$ ion is essential for the substrate cleavage by Cas12i1 (Supplementary Fig. 7b).

\section{Discussion}

In the recently reported cryo-EM and crystal structures of Cas12il and Cas12i2 complexes, the partial target duplex containing the PAM sequences was presented ${ }^{27,28}$. The mechanism by which Cas12i unzips the double-stranded target duplex was short of investigation and information related to the R-loop formation and recognition in Cas12i was almost not reported. To address these questions, we report crystal structures of Cas12i1 ternary complexes in the pre-cleavage and post-cleavage R-loop states. The zipper motif and two groups of "phosphate lock" interactions have been identified to facilitate the target DNA duplex unwinding in Cas12i1. In both Cas12i1 R-loop complexes, the TD strand is unwound from the NTD strand beyond the PAM duplex and forms a 19-bp heteroduplex with the crRNA guide region. In the Cas12il pre-cleavage R-loop complex, the nucleotide of the TD strand swings to interact with that of the NTD strand beyond the 19-bp heteroduplex. In contrast, 28-bp and 26-bp heteroduplexes were previously reported to be accommodated in the Cas12i1 and Cas12i2 ternary complexes, respectively $y^{27,28}$, possibly due to the incomplete states of the target DNA duplexes and longer crRNA guide regions used to prepare the ternary complexes. This implies that the complete Rloop structure in Cas12i will affect the reasonable length of the heteroduplex formed by the TD strand and the crRNA guide region.

Cas12i1 and Cas12i2 share $27.25 \%$ protein sequence identity. The overlay of the Cas12i1 and Cas12i2 ternary complexes clearly shows that two proteins have similar structural folds (Supplementary Fig. 8a-c). The Helical-I domain in Cas12il contains two separate $\alpha$-helix bundles, and their counterparts in Cas12i2 are defined as two independent domains (the Helical-I and Helical-II domains), which is the main difference between domain compositions of Cas12i1 and Cas12i2. Although nucleotide sequences of the crRNA repeat regions in Cas12il and Cas12i2 systems are obviously different, the stem-loop architectures for their crRNA repeat regions resemble each other closely (Supplementary Fig. 8a-e). In the present study, we proved that Cas12il cleaved the NTD strand prior to the TD strand. The NTD strand was successively cleaved 13-15 nucleotides after the PAM duplex, and the TD strand was cleaved 24 nucleotides after the PAM duplex. Cas12i2 was also found to cleave the TD strand at the same position as observed in Cas12il, whereas the NTD strand was primarily cleaved 31 nucleotides after the PAM duplex by Cas12i2 28 . In addition, the post-cleavage R-loop complex of Cas12il provides not only the information of the R-loop structure after the cis-cleavage of the target duplex, but also the information regarding the Cas12il trans-cleavage.

Due to the lack of the PI domain in the previously reported binary complexes of Cas12i1 and Cas12i2, the conformational movement of the PI domain was not observed 27,28 . This study noticed a remarkable "open to closed" conformational transformation of the PI domain upon the target duplex binding in Cas12i1 (Fig. 6c-e). We also found that residues G235 and A236 of the Cas12i1 PI domain played critical roles in the $5^{\prime}-\mathrm{TTN}-3^{\prime}$ PAM determination. In addition, the previous study revealed that the HL motif (also termed the Lid motif) of Cas12il underwent a conformational rearrangement during transition from binary to ternary complexes ${ }^{27}$. We found that both the HL motif and the PAM-distal $\alpha$-helix bundle of the Helical-I domain in Cas12i1 were significantly rearranged so that they no longer obstructed the substrate accessing to the RuvC active site and the Cas12i1 DNase activity was unleashed. Such conformational rearrangement of the Helical-II domain of Cas12i2 was also reported to activate the Cas12i2 DNase activity ${ }^{28}$.

Although Cas12a, Cas12b, Cas12e, and Cas12i possess the REC and NUC bilobed architectures, the composition and domain distribution of these effectors display considerable divergence $20,29-33,37-39,43,44$. Cas12i1 shares most structural features with Cas12b (Supplementary Fig. 1a, b). For instance, the Helical-I domains of Cas12i1 and Cas12b 
a

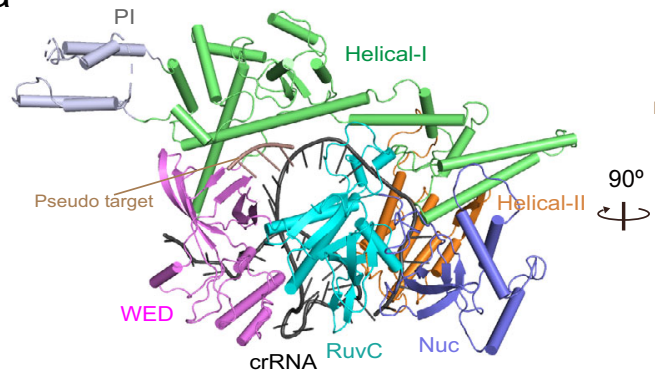

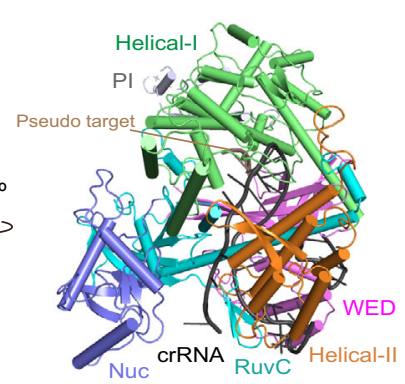

d

b

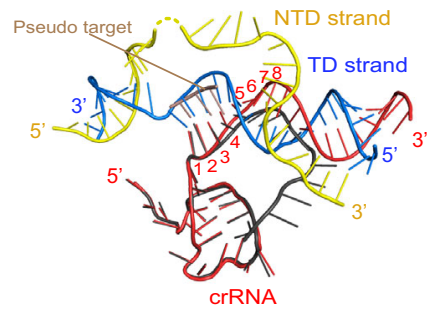

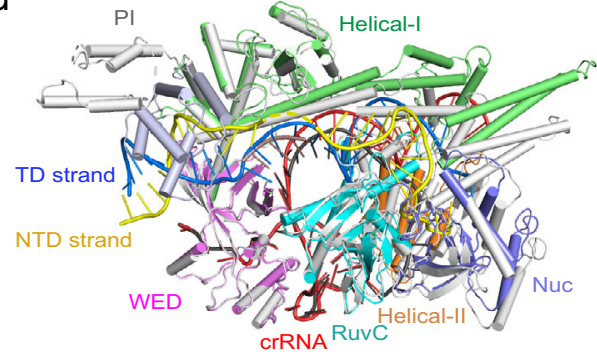

C
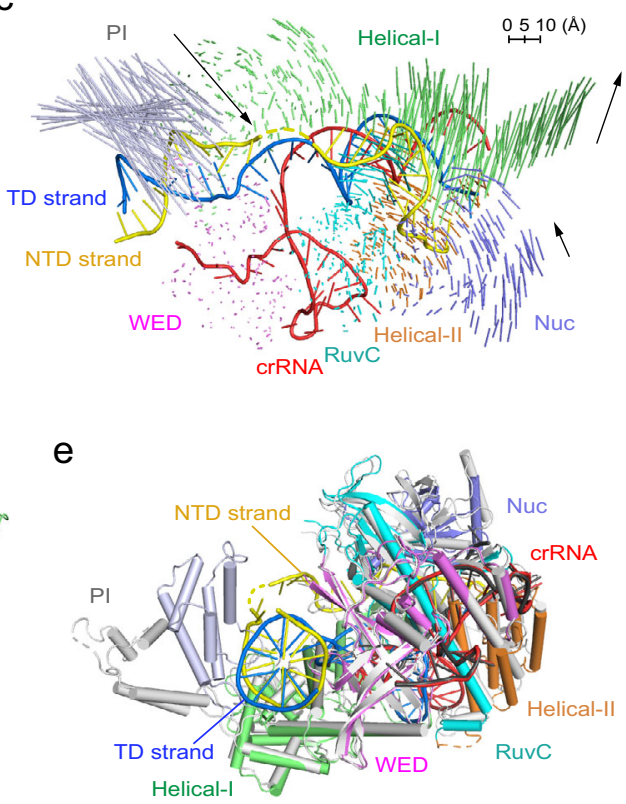

f

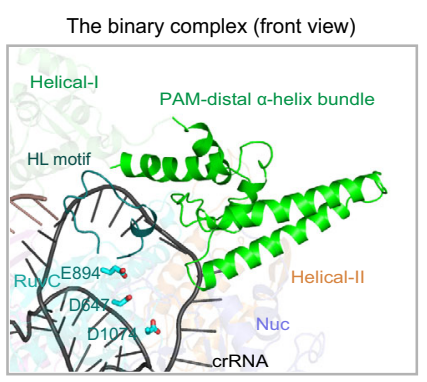

The pre-cleavage R-loop complex (front view)

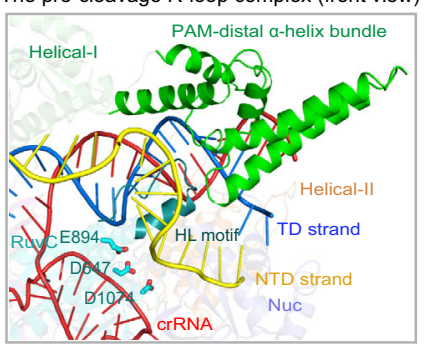

g

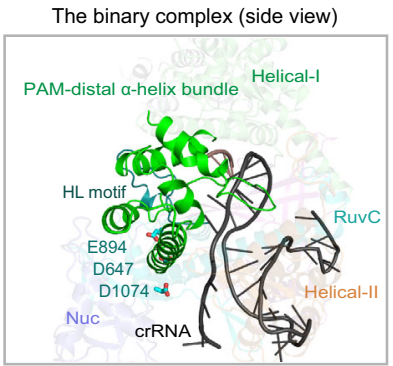

The pre-cleavage R-loop complex (side view)

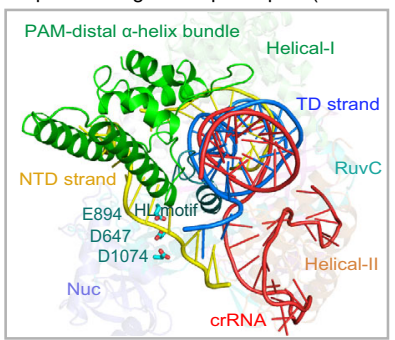

$\mathrm{h}$

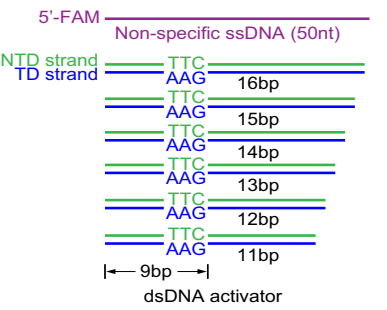

5'-FAM

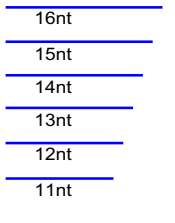

ssDNA activator

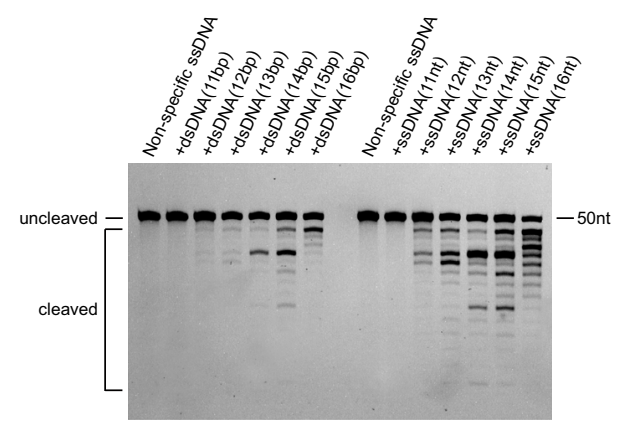

Fig. 6 The target binding triggers the Cas12i1 DNase activity. a Overall structure of the Cas12i1 binary complex shown in two different orientations. b Structural comparison of crRNAs in the Cas12i1 binary and pre-cleavage R-loop complexes. crRNAs are colored as in (a) and Fig. 1. c Structural comparison of Cas12i1 in the binary and pre-cleavage R-loop complexes. Vector length correlates with the motion scale of each domain in Cas12i1. Black arrows indicate domain movements upon the target duplex binding. d, e The overlay of the Cas12i1 binary and pre-cleavage R-loop complexes shown in front and side views. Cas12i1 and crRNA in the binary complex are colored in silver and black, and the pre-cleavage R-loop complex is colored as in Fig. 1. Note that the PI and Helical-I domains undergo significant rearrangements. $\mathbf{f}$ Close-up front views of the Cas12i1 binary and pre-cleavage R-loop complexes. The PAM-distal $\alpha$-helix bundle of the Helical-I domain exhibits an upward movement and the $\mathrm{HL}$ motif rearranges its conformation. $\mathbf{g}$ Close-up side views of the Cas12i1 binary and pre-cleavage R-loop complexes. A single central channel can be observed in the binary complex to accommodate the crRNA guide region, whereas two channels separated by the $\mathrm{HL}$ motif are formed in the pre-cleavage R-loop complex to accommodate the crRNA guide region-TD strand heteroduplex and the NTD strand. $\mathbf{h}$ Schematic representations of double-stranded DNA (dsDNA) activators and single-stranded DNA (ssDNA) activators with different lengths. Denaturing gel demonstrating the cleavage of the fluorescently labeled non-specific ssDNA by wild-type Cas12i1 in complex with crRNA and in the presence of dsDNA or ssDNA activator. The cleavage assays were repeated three times independently to confirm the repeatability and source data are provided as a Source data file.

have a similar structural fold. The Cas12i1 PI domain and the AacCas12b (Alicyclobacillus acidoterrestris Cas12b) counterpart (residues 136-173aa) are connected to the Helical-I domains of the respective effectors. In contrast, the Cas12a PI domain is located on the opposite side of the PAM duplex and is connected to the WED domain (Supplementary Fig. 1c). Cas12e lacks an equivalent of the PI domain, but contains a unique non-target strand-binding (NTSB) domain connected to the Helical-I domain (Supplementary Fig. 1d). Moreover, the Helical-II domains of Cas12i1 and Cas12b are connected to the respective RuvC domains, whereas the corresponding Helical-II domains of Cas12a and Cas12e are connected to the Helical-I and WED domains, but not the RuvC domain (Supplementary Fig. 1a-d). 
In addition, the crRNA repeat regions in the Cas12i1 and Cas12i2 complexes display a simple stem-loop structure, and the one in the Cas12a complex folds into a pseudoknot structure. Single guide RNAs (sgRNAs) in the Cas12b and Cas12e ternary complexes present distinct and complicated architectures.

In Cas12i1, the zipper motif and the phosphate locks facilitate the target duplex unwinding beyond the PAM duplex, after which the preorganized crRNA seed region contributes to base pairing with the unwound TD strand. We can discern a structural motif (residues 108-130aa) of AacCas12b resembling the zipper motif of Cas12i1, implying that AacCas12b may employ similar mechanism to aid in the target duplex unwinding (Supplementary Fig. 1e). In contrast, FnCas12a (Francisella novicida Cas12a) uses a loop-lysine helix-loop (LKL) motif (residues 662-679aa) from its PI domain to promote the target duplex unwinding ${ }^{44}$ (Supplementary Fig. 1f). Cas12e instead uses the unique NTSB domain for this purpose ${ }^{20}$. In addition, the phosphate lock and the preorganized seed region have also been observed in Cas12a and Cas $12 \mathrm{~b}$ to facilitate the target duplex unwinding and promote the heteroduplex formation $29,33,37,43$. Following the initial unzipping of the target duplex, the progressive formation of the crRNA guide region-TD strand heteroduplex can greatly reshape conformation of Cas12 effectors. The 19-bp heteroduplex is presented in two Cas12i1 R-loop complexes. The 20-bp heteroduplex has been found in the ternary complexes of Cas12a and Cas $12 b^{29,33,37}$. Beyond the 20-bp heteroduplex, the TD strand makes a sharp turn and interacts with the NTD strand in Cas $12 \mathrm{a}^{39,43}$.

In the binary complexes, Cas 12 effectors possess a central channel which binds the guide region of their crRNA or sgRNA. Target duplex binding induces significant conformational changes in Cas12 effectors that result in replacement of the crRNA guide region-binding central channel with two separate channels to accommodate the crRNA guide region-TD strand heteroduplex and the unpaired NTD strand. In Cas12a, the PI domain and the REC lobe (including the Helical-I and Helical-II domains) undergo remarkable rearrangements (Supplementary Fig. 9a). The PAM-interacting cleft of Cas12a adjusts from open to closed conformation to bind the target duplex ${ }^{37}$. The Cas12a Helical-II domain detaches from the surface of the RuvC and Nuc domains, leaving the passage to the RuvC active site unobstructed. During the hybridization of the crRNA guide region and the TD strand in Cas12a, certain key motifs such as the finger, helix-loop-helix (HLH), and REC linker from the REC lobe as well as the lid motif in the RuvC domain work concertedly to conformationally activate the DNase activity of Cas $12 \mathrm{a}^{38,44}$. In AacCas12b, while the REC lobe goes through modest rearrangement $^{29}$ (Supplementary Fig. 9b), crucial movement of the HL motif (residues 850-870aa) from the RuvC domain could trigger the DNase activity (Supplementary Fig. 9c). Compared to Cas12a, the PAM-interacting cleft of Cas12il undergoes a greater movement to fulfill the open-to-closed adjustment (Fig. 6c-e). Notably, conformational rearrangements of the PAM-distal $\alpha$ helix bundle from the Helical-I domain and the HL motif from the RuvC domain allow substrates access to the RuvC active site in Cas12i1 (Fig. 6f, g).

All reported Cas12 effectors generate staggered double-strand breaks using the single DNase active site in the RuvC domain. Cas12a and Cas12b cleave the NTD strand at 14 and 17 nucleotides after the PAM, respectively ${ }^{29,44}$. Cas12e and Cas12i1 cut the NTD strand at 12-14 and 13-15 nucleotides after the PAM, respectively ${ }^{20}$. A possible explanation for the successive cleaving of the NTD strand by Cas12i1 is that Cas12il first cleaves 15 nucleotides after the PAM, after which the remainder of the NTD strand is stretched and fed into the RuvC active site for two more consecutive cuts. Afterward, the NTD strand could be further trans-processed by Cas12i1. The phenomenon of the successive cleaving of the NTD strand by Cas12e and Cas12i1 should be taken into account for improving the genomeediting fidelity.

The state of the TD strand loading into the RuvC active site was first observed in Cas $12 b^{29}$. As seen in the Cas $12 b$ ternary complex, direct elongation of the TD strand beyond the heteroduplex would clash with the Helical-II domain of Cas12b (Supplementary Fig. 1b). Consequently, the TD strand is forced to make a reverse turn. Further stabilized and guided by the Nuc domain, the TD strand is loaded into the RuvC catalytic pocket and cleaved at 24 nucleotides after the $\mathrm{PAM}^{29}$. The TSL domain in Cas12e and the Nuc domain in Cas12a are speculated to play an active role in unwinding of the PAM-distal target duplex and loading the TD strand into the RuvC catalytic pocket ${ }^{20,39}$. In the Cas12i1 pre-cleavage R-loop complex, we did not observe clear density for nucleotides beyond the 20th nucleotide of the NTD strand after the PAM. A direct elongation of the $3^{\prime}$-end of the NTD strand will clash with helix $\alpha 2$ of the Helical-II domain, which may disturb the PAM-distal base pairing between the TD and NTD strands (Supplementary Fig. 9d). Therefore, the Cas12i1 Helical-II domain may play a key role in unwinding of two strands in the PAM-distal target duplex and facilitates the TD-strand loading into the RuvC catalytic pocket.

In summary, we determine the structures of the Cas12i1 Rloop complexes prior to and after target DNA cleavage, elucidate the mechanisms for the target duplex unwinding, R-loop formation and cleavage of the NTD and TD strands, reveal the role of domains and key components for the TD strand loading into the RuvC active site, and identify critical residues involved in PAM determination. Moreover, we report the structure of the Cas12i1 binary complex, analyze the conformational changes in Cas12i1 that occur during transition from binary to ternary complex, and explain the mechanisms underlying the conformational activation of the Cas12i1 DNase activity. Based on our structural and biochemical studies, we finally propose a multi-step model for the Cas12i1 activation (Fig. 7). Our study provides mechanistic insights for rational application of the CRISPR-Cas12i system and its further development into a powerful tool for research and therapeutic purposes.

\section{Methods}

Purification of the Cas12i1 binary complex assembled in vivo. The full-length gene encoding Cas12i1 was codon-optimized and synthesized (Supplementary Table 2). The Cas12il gene sequence was amplified by polymerase chain reaction (PCR) and inserted between NdeI and XhoI sites of the pET-30b vector fused with a C-terminal His-tag sequence. CRISPR array template was also designed and synthesized (Supplementary Table 3), after which its gene sequence was cloned into NdeI and XhoI sites of the pCDFDuet-1 vector.

To obtain the Cas12i1 binary complex assembled in vivo, the pET-30b vector encoding the Cas12i1 D647A mutant and the pCDFDuet-1 vector encoding CRISPR template were co-transformed into E. coli Rosetta (DE3) cells (Novagen). The bacterial cultures were grown at $37^{\circ} \mathrm{C}$ for about $3 \mathrm{~h}$ (until $\mathrm{OD}_{600}$ reached 0.6), following which $0.2 \mathrm{mM}$ IPTG was added to induce overexpression at $18{ }^{\circ} \mathrm{C}$ for 12 h. The cells were collected and lysed in buffer A ( $25 \mathrm{mM}$ Tris- $\mathrm{HCl}$ (pH 7.5), 500 $\mathrm{mM} \mathrm{NaCl}, 3.5 \mathrm{mM} \beta$-mercaptoethanol, $1 \mathrm{mM}$ PMSF). In the first step, the binary complex was purified with an Ni-NTA Superflow (QIAGEN) column. The target sample was eluted from the column in buffer A containing gradually increasing concentrations of imidazole. The binary complex sample was further purified with a Heparin HP column (GE Healthcare) using a salt gradient between buffer B1 (25 $\mathrm{mM}$ Tris- $\mathrm{HCl}$ ( $\mathrm{pH} 7.5$ ), $300 \mathrm{mM} \mathrm{NaCl}, 2 \mathrm{mM} \mathrm{MgCl}, 1 \mathrm{mM}$ DTT) and buffer B2 (25 mM Tris- $\mathrm{HCl}$ ( $\mathrm{pH} 7.5), 1.2 \mathrm{M} \mathrm{NaCl}, 2 \mathrm{mM} \mathrm{MgCl}_{2}, 1 \mathrm{mM}$ DTT). Afterward, the target sample was dialyzed against buffer $\mathrm{C}(25 \mathrm{mM}$ Tris- $\mathrm{HCl}(\mathrm{pH} 7.5), 150 \mathrm{mM}$ $\mathrm{NaCl}, 2 \mathrm{mM} \mathrm{MgCl} 2,1 \mathrm{mM} \mathrm{DTT})$ and purified with a Superdex 200 Increase (10/ $300 \mathrm{GL})$ column (GE Healthcare). The purified sample was concentrated and stored at $-80^{\circ} \mathrm{C}$ until further use.

The selenomethionine (SeMet)-labeled Cas12i1 (D647A mutant or wild type) binary complexes were expressed in E. coli Rosetta (DE3) cells grown in M9 minimal medium supplemented with SeMet, Lys, Phe, Thr, Val, Leu, and Ile. The SeMet-labeled Cas12il (D647A mutant or wild type) binary complexes and the 


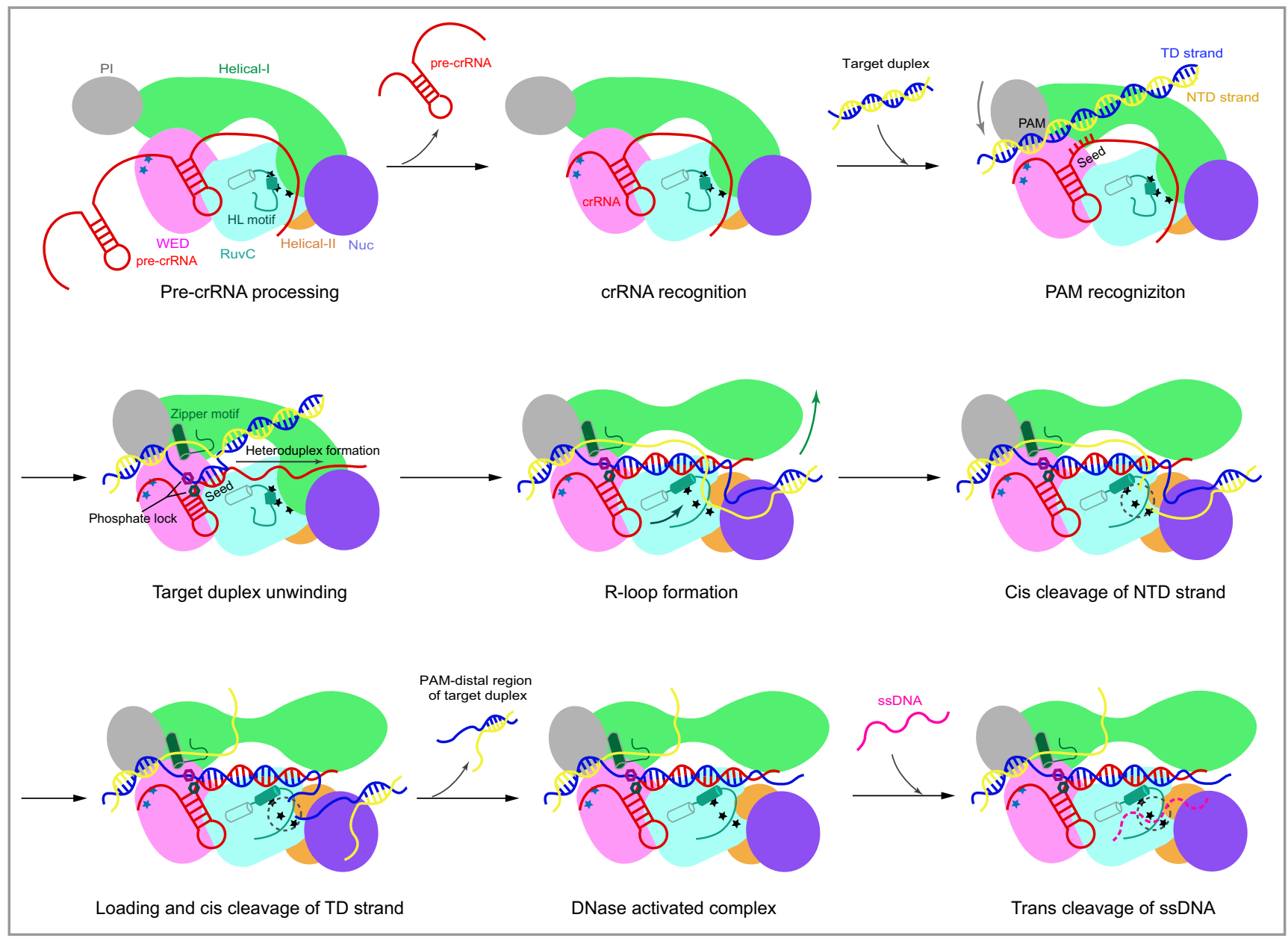

Fig. 7 A multi-step model for the activation of Cas12i1 cis- and trans-cleavage. The apo Cas12i1 recognizes pre-crRNA, processes it into mature crRNA, and forms a Cas12i1-crRNA binary complex. The open PAM-interacting cleft of the binary complex screens the DNA substrate. When matched with a correct PAM, the PAM-interacting cleft of Cas12i1 closes. The zipper motif and the phosphate lock work together to assist unwinding of the target duplex beyond the PAM, and the unwound TD strand is directed toward the preorganized crRNA seed region for interrogation. Correct matching with the crRNA seed region further advances the 19-bp heteroduplex formation, which concomitantly drives conformational rearrangements in Cas12i1. The Helical-I domain and the $\mathrm{HL}$ motif are released from the RuvC catalytic pocket to participate in forming of two parallel channels that accommodate the heteroduplex and the unpaired NTD strand, respectively. As a result, the R-loop is formed and the Cas12i1 DNase activity is conformationally activated. Meanwhile, the NTD strand is guided to the RuvC catalytic pocket and cleaved. The Helical-II and Nuc domains then promote unwinding of the PAM-distal target DNA duplex and loading of the TD strand into the RuvC catalytic pocket, following which the TD strand is cleaved and the PAM-distal cleavage product is released from the complex. The activated Cas12i1 could cleave any available single-stranded DNA (ssDNA) in trans.

unlabeled Cas12i1 (wild type) binary complex were obtained following the protocol described above.

Formation of the Cas12i1 ternary complex. Gel purified 40-nt target strand and non-target strand of target DNA duplex were purchased and dissolved in buffer D ( $25 \mathrm{mM}$ Tris- $\mathrm{HCl}$ (pH 7.5), $100 \mathrm{mM} \mathrm{NaCl}, 2 \mathrm{mM} \mathrm{MgCl}_{2}$ ) to form a $100 \mathrm{mM}$ stock. The two strands were then mixed at a molar ratio of $1: 1$, denatured at $95^{\circ} \mathrm{C}$ for 5 min, and annealed by slow cooling down to room temperature within $1 \mathrm{~h}$. The prepared binary complex and the annealed target duplex were mixed together in buffer $\mathrm{C}$ at an approximate molar ratio of 1:1.2 and incubated at $16^{\circ} \mathrm{C}$ for $1 \mathrm{~h}$. Following gel-filtration purification with a Superdex 200 Increase (10/300 GL) column (GE Healthcare), fractions containing ternary complex were collected, concentrated to $\mathrm{A}_{280}=12.0$, and used for further experiments.

Purification of the uncomplexed Cas12i1. The wild-type Cas12i1 or its mutant encoded by the pET-30b vector was expressed in E. coli Rosetta (DE3) cells (Novagen). After culturing and protein expression, the cells were lysed in buffer A and Cas12il was purified using the protocol described for the purification of the binary complex, with the exception that buffers B1, B2, and C did not contain 2 $\mathrm{mM} \mathrm{MgCl} 2$. The purified Cas12il samples were concentrated and stored for later use in the cleavage assays.
In vitro synthesis and purification of crRNA. crRNA sequence was synthesized and purified following the previous protocol ${ }^{45,46}$ (Supplementary Table 3). In brief, DNA fragment encoding crRNA was inserted between StuI and HindIII sites of a modified pUC-119 vector. The vector was amplified in E. coli DH5a cells, extracted, linearized by HindIII, and purified. In vitro transcription was carried out using homemade T7 RNA polymerase and the reaction sample was resolved using a $20 \%$ denaturing ( $8 \mathrm{M}$ urea) polyacrylamide gel. The target RNA band was excised and eluted using the Elutrap system (GE Healthcare). The eluted RNA sample was concentrated and de-salted by ethanol precipitation, after which it was dissolved in DEPC-treated $\mathrm{H}_{2} \mathrm{O}$ and stored at $-80^{\circ} \mathrm{C}$.

Crystallization. The SeMet-labeled and unlabeled Cas12i1 D647A mutant ternary complexes were crystallized using the hanging-drop vapor diffusion method at $16^{\circ}$ C. Crystals were acquired by mixing $1 \mu \mathrm{l}$ of complex solution $\left(\mathrm{A}_{280 \mathrm{~nm}}=12.0\right)$ and $1 \mu \mathrm{l}$ of reservoir solution $(0.1 \mathrm{M}$ sodium citrate $(\mathrm{pH}$ 5.6), $17 \%(\mathrm{w} / \mathrm{v})$ Polyethylene glycol 3350, and $0.1 \mathrm{M}$ sodium citrate tribasic dihydrate). Crystals grew to full size within 12 days, and were then harvested and cryoprotected in reservoir solution supplemented with $16 \%(\mathrm{v} / \mathrm{v})$ glycerol and flash-frozen in liquid nitrogen.

The wild-type Cas12i1 ternary complex was crystallized using the method described above with the reservoir solution adjusted to $0.1 \mathrm{M}$ sodium citrate $(\mathrm{pH}$ 5.4), 15\% (w/v) Polyethylene glycol 3350, and 0.2 M lithium chloride. Crystals grew to full size within 9 days and were cryoprotected in reservoir solution added with $18 \%(\mathrm{v} / \mathrm{v})$ glycerol and flash-cooled. 
The SeMet-labeled Cas12i1 (wild type) binary complex assembled in vivo was crystallized by the hanging-drop vapor diffusion method at $16^{\circ} \mathrm{C}$. Crystals were obtained by mixing $1 \mu \mathrm{l}$ of complex solution $\left(\mathrm{A}_{280 \mathrm{~nm}}=12.3\right)$ and $1 \mu \mathrm{l}$ of reservoir solution (0.1 M sodium citrate (pH 5.2), $17 \%$ (w/v) Polyethylene glycol $3350,2 \%$ Tacsimate ( $\mathrm{pH} 4.0$ ), and $0.15 \mathrm{M}$ sodium citrate tribasic dihydrate). Crystals grew to full size within 10 days and were harvested, cryoprotected in reservoir solution containing $16 \%(\mathrm{v} / \mathrm{v})$ glycerol and flash-cooled.

Data collection and structure determination. X-ray diffraction datasets were collected on beamline BL-17U1 of Shanghai Synchrotron Radiation Facility (SSRF) by using the program Blu-Ice and the X-ray wavelength $0.9792 \AA$. Datasets were automatically processed by the program XDS and XIA2 integrated into data collection platform of the beamline $e^{47,48}$, and scaled by the program AIMLESS in CCP $4^{49,50}$. The crystal structure of the SeMet-labeled Cas12i1 (D647A mutant) ternary complexes was solved by single-wavelength anomalous dispersion (SAD) method using the program AutoSol in PHENIX ${ }^{51}$. Model building was completed in COOT ${ }^{52}$. Model refinement was performed with REFMAC in CCP4 format ${ }^{53}$. Based on the SeMet-labeled model of the ternary complex, crystal structures of the unlabeled Cas12i1 (D647A mutant or wild type) ternary complex and the wild-type Cas12il binary complex were solved by the molecular replacement (MR) method using the program Phaser-MR in PHENIX ${ }^{51}$. Asymmetric units in four kinds of crystals all contain single copy of the ternary or binary complex. Figures of structures were prepared with PyMOL (http://pymol.org). Detailed data collection and refinement statistics were summarized in Supplementary Table 1.

Site-directed mutagenesis. Vector encoding the wild-type Cas12i1 was used as a template and oligonucleotides containing the desired mutations were designed (Supplementary Table 4). PCR reactions were carried out to accomplish the sitedirected mutagenesis and results were verified by DNA sequencing.

Plasmid cleavage assay. Synthetic oligonucleotides (Supplementary Table 5) encoding two strands of target DNA duplex were annealed and inserted between EcoRI and HindIII sites of the pUC-19 vector. Plasmids were verified by DNA sequencing and further linearized by ScaI. Reaction mixtures were prepared by incubating Cas12i1 (final concentration of $0.33 \mu \mathrm{M}$ ) with crRNA (final concentration of $0.33 \mu \mathrm{M}$ ) to form the binary complex at $25^{\circ} \mathrm{C}$ for $30 \mathrm{~min}$ in assay buffer (50 mM Tris- $\mathrm{HCl}$ ( $\mathrm{pH} 8.0$ ), $150 \mathrm{mM} \mathrm{NaCl}, 10 \mathrm{mM} \mathrm{MgCl} 2,1 \mathrm{mM}$ DTT). The cleavage assays were then carried out by adding $1000 \mathrm{ng}$ pUC- 19 target plasmids at $45^{\circ} \mathrm{C}$ for $30 \mathrm{~min}$ in reaction mixtures with total volume adjusted to $30 \mu \mathrm{l}$. Each cleavage assay was repeated three times independently to confirm the repeatability of the results. Reactions were terminated by adding EDTA and Proteinase $\mathrm{K}$ to final concentrations of $100 \mathrm{mM}$ and $0.8 \mathrm{mg} / \mathrm{ml}$ at $37^{\circ} \mathrm{C}$ for $30 \mathrm{~min}$, respectively. Reaction samples were analyzed by running $1 \%$ agarose gels stained with Gel Stain (Transgene Biotech).

Oligonucleotide cleavage assay. Target and non-target DNA strands carrying $5^{\prime}$ 6FAM and 3'-6FAM fluorescent labels were purchased from Sangon Biotech (Supplementary Table 5) and annealed to form a $70 \mu \mathrm{M}$ stock. To prepare the cleavage assays, Cas12il and crRNA (both with a final concentration of $10 \mu \mathrm{M}$ ) were incubated to form the binary complex at $25^{\circ} \mathrm{C}$ for $30 \mathrm{~min}$ in assay buffer $(50$ $\mathrm{mM}$ Tris- $\mathrm{HCl}$ ( $\mathrm{pH} 8.0$ ), $150 \mathrm{mM} \mathrm{NaCl}, 2 \mathrm{mM} \mathrm{MgCl} 2,1 \mathrm{mM} \mathrm{DTT}$ ). The cleavage assays were performed at $37^{\circ} \mathrm{C}$ for $30 \mathrm{~min}$ by adding fluorescently labeled doublestranded substrates (final concentration of $5 \mu \mathrm{M}$ ) in reaction mixtures with total volume adjusted to $20 \mu \mathrm{l}$. The cleavage assays were repeated independently three times. Reactions were terminated by adding EDTA and Proteinase $\mathrm{K}$ to final concentrations of $100 \mathrm{mM}$ and $0.8 \mathrm{mg} / \mathrm{ml}$ at $37^{\circ} \mathrm{C}$ for $30 \mathrm{~min}$, respectively. Reaction samples were run on a $20 \%$ PAGE TBE-urea denaturing gel and the cleavage results were visualized using BIO-RAD Universal Hood II System.

Cas12i1 activation and trans-cleavage assay. Double-stranded DNA (dsDNA) activators containing 11-16-bp sequences after the PAM duplex and 11-16-nt single-stranded DNA (ssDNA) activators were tested for their ability to trigger the Cas12il DNase activity (Supplementary Table 5). A non-specific ssDNA carrying a $5^{\prime}$-6FAM fluorescent label was used as the substrate for the trans-cleavage assay (Supplementary Table 5). The Cas12i1 binary complex used in the assay was prepared as described in the protocol for oligonucleotide cleavage assay. The transcleavage assays were carried out by adding dsDNA or ssDNA activator with a final concentration of $5 \mu \mathrm{M}$ and fluorescently labeled non-specific ssDNA with a final concentration of $5 \mu \mathrm{M}$. The assays were performed at $37^{\circ} \mathrm{C}$ for $60 \mathrm{~min}$ in reaction mixtures with total volume adjusted to $20 \mu \mathrm{l}$. All cleavage assays were repeated independently three times. Reactions were terminated by adding EDTA and Proteinase $\mathrm{K}$ to final concentrations of $100 \mathrm{mM}$ and $0.8 \mathrm{mg} / \mathrm{ml}$ at $37^{\circ} \mathrm{C}$ for $30 \mathrm{~min}$, respectively. Reaction samples were run on a $20 \%$ PAGE TBE-urea denaturing gel and results were visualized as using BIO-RAD Universal Hood II System.
Reporting summary. Further information on research design is available in the Nature Research Reporting Summary linked to this article.

\section{Data availability}

The atomic coordinates and structure factors of the Cas12il pre-cleavage R-loop complex, post-cleavage R-loop complex, and binary complex have been deposited in the Protein Data Bank under the accession codes 7D2L, 7D3J, and 7D8C. Source data are provided with this paper. Other data are available from the corresponding author upon reasonable request.

Received: 7 January 2021; Accepted: 20 May 2021;

Published online: 09 June 2021

\section{References}

1. Van der Oost, J., Westra, E. R., Jackson, R. N. \& Wiedenheft, B. Unravelling the structural and mechanistic basis of CRISPR-Cas systems. Nat. Rev. Microbiol. 12, 479-492 (2014).

2. Koonin, E. V., Makarova, K. S. \& Wolf, Y. I. Evolutionary genomics of defense systems in archaea and bacteria. Annu. Rev. Microbiol. 71, 233-261 (2017).

3. Hille, F. et al. The biology of CRISPR-Cas: backward and forward. Cell 172, 1239-1259 (2018)

4. Amitai, G. \& Sorek, R. CRISPR-Cas adaptation: insights into the mechanism of action. Nat. Rev. Microbiol. 14, 67-76 (2016).

5. Knott, G. J. \& Doudna, J. A. CRISPR-Cas guides the future of genetic engineering. Science 361, 866-869 (2018).

6. Koonin, E. V., Makarova, K. S. \& Zhang, F. Diversity, classification and evolution of CRISPR-Cas systems. Curr. Opin. Microbiol. 37, 67-78 (2017).

7. Shmakov, S. et al. Diversity and evolution of class 2 CRISPR-Cas systems. Nat. Rev. Microbiol. 15, 169-182 (2017).

8. Makarova, K. S. et al. Evolutionary classification of CRISPR-Cas systems: a burst of class 2 and derived variants. Nat. Rev. Microbiol. 18, 67-83 (2020).

9. Jinek, $M$. et al. A programmable dual-RNA-guided DNA endonuclease in adaptive bacterial immunity. Science 337, 816-821 (2012).

10. Cong, L. et al. Multiplex genome engineering using CRISPR/Cas systems. Science 339, 819-823 (2013)

11. Hsu, P. D., Lander, E. S. \& Zhang, F. Development and applications of CRISPR-Cas9 for genome engineering. Cell 157, 1262-1278 (2014).

12. Wang, H., La Russa, M. \& Qi, L. S. CRISPR/Cas9 in genome editing and beyond. Annu. Rev. Biochem. 85, 227-264 (2016).

13. Wright, A. V., Nunez, J. K. \& Doudna, J. A. Biology and applications of CRISPR systems: harnessing nature's toolbox for genome engineering. Cell 164, 29-44 (2016).

14. Jiang, F. \& Doudna, J. A. CRISPR-Cas9 structures and mechanisms. Annu. Rev. Biophys. 46, 505-529 (2017).

15. Zetsche, B. et al. Cpf1 is a single RNA-guided endonuclease of a class 2 CRISPR-Cas system. Cell 163, 759-771 (2015).

16. Kim, D. et al. Genome-wide analysis reveals specificities of Cpf1 endonucleases in human cells. Nat. Biotechnol. 34, 863-868 (2016).

17. Kleinstiver, B. P. et al. Genome-wide specificities of CRISPR-Cas Cpf1 nucleases in human cells. Nat. Biotechnol. 34, 869-874 (2016).

18. Teng, F. et al. Repurposing CRISPR-Cas12b for mammalian genome engineering. Cell Disco. 4, 63 (2018).

19. Strecker, J. et al. Engineering of CRISPR-Cas12b for human genome editing Nat. Commun. 10, 212 (2019)

20. Liu, J. J. et al. CasX enzymes comprise a distinct family of RNA-guided genome editors. Nature 566, 218-223 (2019).

21. Harrington, L. B. et al. Programmed DNA destruction by miniature CRISPRCas14 enzymes. Science 362, 839-842 (2018).

22. Yan, W. X. et al. Functionally diverse type V CRISPR-Cas systems. Science 363, 88-91 (2019)

23. Harrington, L. B. et al. A scoutRNA is required for some type V CRISPR-Cas systems. Mol. Cell 79, 416-424 (2020). e5.

24. Pausch, P. et al. CRISPR-CasPhi from huge phages is a hypercompact genome editor. Science 369, 333-337 (2020).

25. Ran, F. A. et al. Double nicking by RNA-guided CRISPR Cas9 for enhanced genome editing specificity. Cell 154, 1380-1389 (2013).

26. Shmakov, S. et al. Discovery and functional characterization of diverse class 2 CRISPR-Cas systems. Mol. Cell 60, 385-397 (2015).

27. Zhang, H., Li, Z., Xiao, R. \& Chang, L. Mechanisms for target recognition and cleavage by the Cas12i RNA-guided endonuclease. Nat. Struct. Mol. Biol. 27, 1069-1076 (2020).

28. Huang, X. et al. Structural basis for two metal-ion catalysis of DNA cleavage by Cas12i2. Nat. Commun. 11, 5241 (2020). 
29. Yang, H., Gao, P., Rajashankar, K. R. \& Patel, D. J. PAM-dependent target DNA recognition and cleavage by C2c1 CRISPR-Cas endonuclease. Cell 167, 1814-1828 (2016).

30. Liu, L. et al. C2c1-sgRNA complex structure reveals RNA-guided DNA cleavage mechanism. Mol. Cell 65, 310-322 (2017).

31. Wu, D., Guan, X., Zhu, Y., Ren, K. \& Huang, Z. Structural basis of stringent PAM recognition by CRISPR-C2c1 in complex with sgRNA. Cell Res. 27, 705-708 (2017).

32. Dong, D. et al. The crystal structure of Cpf1 in complex with CRISPR RNA. Nature 532, 522-526 (2016).

33. Yamano, T. et al. Crystal structure of Cpf1 in complex with guide RNA and target DNA. Cell 165, 949-962 (2016).

34. Anders, C., Niewoehner, O., Duerst, A. \& Jinek, M. Structural basis of PAMdependent target DNA recognition by the Cas9 endonuclease. Nature 513, 569-573 (2014).

35. Nishimasu, H. et al. Crystal structure of Cas9 in complex with guide RNA and target DNA. Cell 156, 935-949 (2014).

36. Jiang, F. et al. Structures of a CRISPR-Cas9 R-loop complex primed for DNA cleavage. Science 351, 867-871 (2016).

37. Gao, P., Yang, H., Rajashankar, K. R., Huang, Z. \& Patel, D. J. Type V CRISPR-Cas Cpf1 endonuclease employs a unique mechanism for crRNAmediated target DNA recognition. Cell Res. 26, 901-913 (2016).

38. Stella, S. et al. Conformational activation promotes CRISPR-Cas12a catalysis and resetting of the endonuclease activity. Cell 175, 1856-1871 (2018). e21.

39. Swarts, D. C. \& Jinek, M. Mechanistic Insights into the cis- and trans-Acting DNase Activities of Cas12a. Mol. Cell 73, 589-600 (2019).

40. Goedken, E. R. \& Marqusee, S. Co-crystal of Escherichia coli RNase HI with $\mathrm{Mn}^{2+}$ ions reveals two divalent metals bound in the active site. J. Biol. Chem. 276, 7266-7271 (2001).

41. Nowotny, M., Gaidamakov, S. A., Crouch, R. J. \& Yang, W. Crystal structures of RNase $\mathrm{H}$ bound to an RNA/DNA hybrid: substrate specificity and metaldependent catalysis. Cell 121, 1005-1016 (2005).

42. Jiang, F., Zhou, K., Ma, L., Gressel, S. \& Doudna, J. A. A Cas9-guide RNA complex preorganized for target DNA recognition. Science 348, 1477-1481 (2015).

43. Swarts, D. C., van der Oost, J. \& Jinek, M. Structural basis for guide RNA processing and seed-dependent DNA targeting by CRISPR-Cas12a. Mol. Cell 66, 221-233 (2017). e4

44. Stella, S., Alcon, P. \& Montoya, G. Structure of the Cpf1 endonuclease R-loop complex after target DNA cleavage. Nature 546, 559-563 (2017).

45. Serganov, A. et al. Ribosomal protein S15 from Thermus thermophilus-cloning, sequencing, overexpression of the gene and RNAbinding properties of the protein. Eur. J. Biochem. 246, 291-300 (1997).

46. Zhang, B. et al. Structural insights into Cas13b-guided CRISPR RNA maturation and recognition. Cell Res. 28, 1198-1201 (2018).

47. Kabsch, W. Xds. Acta Crystallogr D. Biol. Crystallogr 66, 125-132 (2010).

48. Winter, G., Lobley, C. M. \& Prince, S. M. Decision making in xia2. Acta Crystallogr D. Biol. Crystallogr 69, 1260-1273 (2013).

49. Evans, P. R. \& Murshudov, G. N. How good are my data and what is the resolution? Acta Crystallogr D. Biol. Crystallogr 69, 1204-1214 (2013).

50. Winn, M. D. et al. Overview of the CCP4 suite and current developments. Acta Crystallogr D. Biol. Crystallogr 67, 235-242 (2011).

51. Adams, P. D. et al. PHENIX: a comprehensive Python-based system for macromolecular structure solution. Acta Crystallogr D. Biol. Crystallogr 66, 213-221 (2010).

52. Emsley, P. \& Cowtan, K. Coot: model-building tools for molecular graphics. Acta Crystallogr D. Biol. Crystallogr 60, 2126-2132 (2004).
53. Vagin, A. A. et al. REFMAC5 dictionary: organization of prior chemical knowledge and guidelines for its use. Acta Crystallogr D. Biol. Crystallogr 60 2184-2195 (2004).

\section{Acknowledgements}

This work was supported by the National Nature Science Foundation of China grants (31770948), the Special Open Fund of Key Laboratory of Experimental Marine Biology, Chinese Academy of Sciences (SKF2020NO1), Marine Economic Development Special Fund of Fujian Province (FJHJF-L-2020-2), the Fujian Provincial Department of Science and Technology (2020Y4007 and 2021H0004), and the High-level personnel introduction grant of Fujian Normal University (Z0210509). The diffraction data were collected at the beamline BL-17U1 of Shanghai Synchrotron Radiation Facility (SSRF).

\section{Author contributions}

B.Z. and S.O. designed the experiments. S.O. and B.Z. supervised the study. B.Z., D.L., and Y.L. prepared Cas12il, its mutants, and the Cas12il complexes. B.Z., V.P., and Y.Y. prepared the in vitro transcribed RNAs. Y.L., D.L., and B.Z. performed the cleavage assays. B.Z., D.L., and Y.L. performed the crystallization screening and optimized the crystallization condition. B.Z. collected X-ray diffraction data and solved the crystal structure. B.Z. wrote the manuscript and prepared the figures. J.C. and J.L. helped with preparing some figures. S.O. and V.P. revised the manuscript.

\section{Competing interests}

The authors declare no competing interests.

\section{Additional information}

Supplementary information The online version contains supplementary material available at https://doi.org/10.1038/s41467-021-23876-5.

Correspondence and requests for materials should be addressed to S.O

Peer review information Nature Communications thanks Jack A. Dunkle and other, anonymous reviewers for their contributions to the peer review of this work. Peer review reports are available.

Reprints and permission information is available at http://www.nature.com/reprints

Publisher's note Springer Nature remains neutral with regard to jurisdictional claims in published maps and institutional affiliations.

Open Access This article is licensed under a Creative Commons Attribution 4.0 International License, which permits use, sharing, adaptation, distribution and reproduction in any medium or format, as long as you give appropriate credit to the original author(s) and the source, provide a link to the Creative Commons license, and indicate if changes were made. The images or other third party material in this article are included in the article's Creative Commons license, unless indicated otherwise in a credit line to the material. If material is not included in the article's Creative Commons license and your intended use is not permitted by statutory regulation or exceeds the permitted use, you will need to obtain permission directly from the copyright holder. To view a copy of this license, visit http://creativecommons.org/ licenses/by/4.0/

(C) The Author(s) 2021 